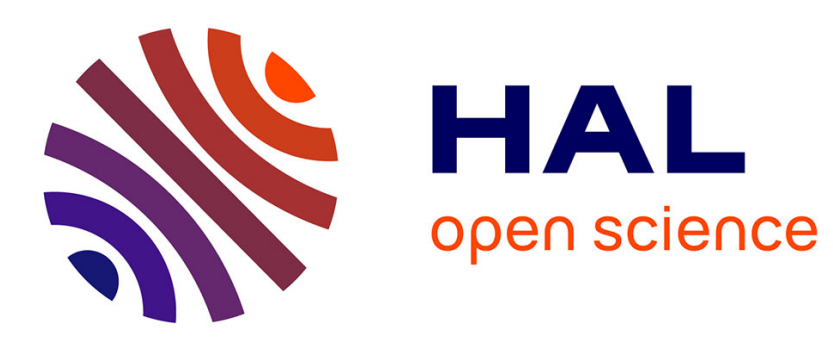

\title{
Premier bilan de la tarification à l'activité (T2A) sur la variabilité des coûts hospitaliers
}

Carine Milcent

\section{To cite this version:}

Carine Milcent. Premier bilan de la tarification à l'activité (T2A) sur la variabilité des coûts hospitaliers. Economie et Prévision, 2017, 210, pp.45 - 67. halshs-01522581

\section{HAL Id: halshs-01522581 https://shs.hal.science/halshs-01522581}

Submitted on 19 Dec 2018

HAL is a multi-disciplinary open access archive for the deposit and dissemination of scientific research documents, whether they are published or not. The documents may come from teaching and research institutions in France or abroad, or from public or private research centers.
L'archive ouverte pluridisciplinaire HAL, est destinée au dépôt et à la diffusion de documents scientifiques de niveau recherche, publiés ou non, émanant des établissements d'enseignement et de recherche français ou étrangers, des laboratoires publics ou privés. 


\title{
Premier bilan de la T2A sur la variabilité des coûts hospitaliers ${ }^{1}$
}

\author{
Carine Milcent \\ CNRS \\ (PSE et CEFC)
}

Résumé : Ce papier étudie la variabilité des coûts hospitaliers pour des séjours comparables en pathologies et en procédures (GHM). A l'époque du budget global, une forte variabilité des coûts était observée entre les hôpitaux publics français. Qu'en est-il aujourd'hui ? Théoriquement, la T2A conduit les établissements à minimiser leurs coûts pour gagner la différence entre le forfait et le coût. Nous montrons une certaine homogénéisation des coûts et à une réelle prise en compte de l'hétérogénéité des individus. Les forfaits par GHM ne capturent cependant pas toute l'hétérogénéité entre les établissements, ni entre les patients. Ainsi, les effets néfastes de sélection des patients ou de diminution du niveau de qualité ne sont pas évités par les forfaits actuels.

Mots clés : T2A, Variabilité des coûts hospitaliers, Groupe Homogène de Malade/Séjours (GHM/GHS), Hétérogénéité des pratiques, Concurrence

Classification JEL : C23, H51, I18

\footnotetext{
${ }^{1}$ Je remercie les membres de l'Agence Technique de l'information sur l'hospitalisation pour leur aide technique et leur grande disponibilité, les participants du séminaire ATIH/ENC. Je remercie tout particulèrement T. Serrurier ainsi que N. Belorgey et A. Aroquiame pour leurs commentaires constructifs et leurs apports.
} 


\title{
French DRG-based payment: First feedbacks on cost variability
}

\author{
Carine Milcent \\ CNRS \\ (PSE and CEFC)
}

\begin{abstract}
In this paper, I study the variability in hospital costs for stays comparable in pathology and procedures (DRG). During the period where hospitals were reimbursed with a global budget, high costs variability was observed. What can we say today? The French DRG-based payment reform (T2A) was implemented from 2004 to 2008. Theoretically, a fee payment leads the supplier to minimize the difference between the fee and the cost. I show that the reform indeed, leads to reduce the cost variability. However, the DRG-based payment still fails to capture part of the sources of hospital heterogeneity and part of the sources of patient heterogeneity. So far, this payment system does not prevent from patient's selection and a negative impact on quality level.
\end{abstract}

Keywords: French-DRG (Diagnosis Related Groups), Variability in hospital costs, DRGbased payment, behaviors' heterogeneity, Competition, Hospitals

JEL classification: C23, H51, I18 


\section{Premier bilan de la T2A sur la variabilité des coûts hospitaliers²}

\section{Résumé Long}

La part des dépenses de santé en France représente 12\% du Produit Intérieur Brut (PIB). Elle se situe après les Etats-Unis et le Pays-Bas. Cette part est très proche d'autres pays tels que l'Allemagne. Les hôpitaux représentent une grande part des dépenses. Toutefois, il s'agit de la part la plus maîtrisée. Les hôpitaux ne représentent plus que 36\% des dépenses de santé en France, contre 43\% en 1983. Ainsi, oui l'hôpital coûte cher mais de moins en moins cher.

Le régulateur doit encourager les hôpitaux à répondre aux besoins, sans induire d'activité injustifiée. Il doit inciter les établissements à l'efficience, c'est à dire à fournir les soins au moindre coût, sans sacrifier la qualité ni sélectionner les patients. Enfin, le régulateur doit distribuer les ressources de manière équitable entre les hôpitaux et, dans cette perspective, être capable d'évaluer l'activité de production de soins des établissements et les contraintes spécifiques auxquelles certains d'entre eux peuvent être confrontés. La Tarification à l'Activité (T2A), introduite en 2004, vise à améliorer l'efficacité de la dépense pour ce poste majeur, les soins hospitaliers. La mise en œuvre du nouveau paiement a été progressive, avec une application intégrale à partir de 2008 pour l'ensemble des hôpitaux.

Dans quelle mesure la mise en place d'une politique favorisant la concurrence entre les établissements a eu un effet sur la variabilité des coûts hospitaliers ? Des premiers travaux sur données françaises portant sur les années 1994 et 1995 étudiait l'effet potentiel d'un passage à la T2A. Il apparaissait de fortes hétérogénéités de coûts entre les séjours d'un même GHM. Il était à craindre que la T2A ait des effets pervers sur la qualité des pratiques des soins.

Depuis, cette période, les établissements de santé ont connu trois changements majeurs : i) la mise en place d'une tarification basée sur un forfait par séjour; ii) une forte augmentation du nombre de groupe de GHM ; iii) l'introduction de la concurrence entre les

\footnotetext{
${ }^{2}$ Je remercie les membres de l'Agence Technique de l'information sur l'hospitalisation pour leur aide technique et leur grande disponibilité, les participants du séminaire ATIH/ENC. Je remercie tout particulèrement T. Serrurier ainsi que N. Belorgey et A. Aroquiame pour leurs commentaires constructifs et leurs apports.
} 
établissements. Par ailleurs, la T2A étant un mécanisme à enveloppe fermée, le montant du forfait par séjour dépend de l'activité globale réalisée par l'ensemble des établissements de santé sur le territoire.

Les données sont issues de la base de l'Etude Nationale de Coûts. La base de coût a été constituée dans le cadre du Programme de médicalisation des systèmes d'informations (PMSI). Les échantillons constitués portent sur des séjours hospitaliers pour infarctus aigu du myocarde dans les hôpitaux publics et privés. Les données du PMSI permettent d'accéder à une information d'une richesse considérable: les observations sont effectuées au niveau de chaque séjour et sont très détaillées en ce qui concerne les diagnostics, les traitements et les coûts associés.

Que pouvons-nous dire aujourd'hui ? Quel a été l'effet de la mise en place de la T2A sur l'hétérogénéité de coût des hôpitaux ? Des patients? Les changements de classification des GHM ont-ils permis d'établir des forfaits plus proches des coûts ?

Ce papier est organisé autour de deux questions que nous nous proposons de reprendre ici.

Est-ce que la classification capture suffisamment l'hétérogénéité des coûts ? Les GHM ne captent qu'un tiers de la variance de coûts. Les hôpitaux étant rémunérés par forfait par GHM, ce résultat soulève la question des conséquences de la prise en compte imparfaite des coûts par les forfaits. Les effets néfastes de sélection des patients ou de diminution du niveau de qualité que peut engendrer une tarification par forfait peuvent apparaître avec les forfaits actuels.

Sans prise en compte des GHM, 43\% de la variance des coûts sont expliquées par les caractéristiques individuelles des patients, les diagnostics et les procédures. Ainsi, les caractéristiques observables des séjours des patients expliquent davantage que la classification des GHM.

Par ailleurs, suivant le statut de l'établissement, les coûts sont plus ou moins homogènes. Quelle que soit l'année considéré, les établissements privés présentent moins de différences de coûts que les autres statuts d'établissements. La comparaison temporelle suggère même un trend de plus grande homogénéisation des coûts par GHM pour les établissements privés que 
pour les autres statuts d'établissements. Ce résultat peut être dû à plus d'efficacité, d'homogénéité des pratiques ou d'homogénéité des caractéristiques des patients. Les données actuelles ne permettent pas de répondre plus avant à cette question.

La seconde série de questions soulevée dans ce papier est : quelle est la part explicative de la variance des coûts due aux écarts permanents de coûts moyens entre les établissements ? Nous montrons que les spécificités permanentes des hôpitaux expliquent environ $20 \%$ de la variance de coût du séjour. Après prise en compte de la classification des GHM ainsi que des caractéristiques des patients, ces spécificités expliquent encore $5 \%$ de la variance totale des coûts. Cette part de variance ne semble pas être due à des différences de durée de séjours. 


\section{Introduction}

La récente réforme de tarification à l'activité, porte sur le mode de remboursement des établissements de santé. Elle introduit un paiement forfaitaire par séjour. Chaque séjour est affecté à un groupe dit Groupe Homogène de Malade (GHM). Ce groupe définit une certaine quantité de soins et un coût moyen c'est à dire une certaine activité hospitalière. Le forfait par séjour correspond-il à un coût homogène quel que soit le patient, quel que soit l'hôpital ? C'est ce que nous étudions ici. S'il apparaissait de fortes variabilités de coûts entre les séjours d'un même GHM, notamment par une forte hétérogénéité des patients et une hétérogénéité des hôpitaux, ceci remettrait alors en question la validité de l'homogénéité de coûts au sein de chaque groupe (variance intra-GHM des coûts). Il serait alors à craindre que la mise en place d'une tarification par forfait par séjour ait des effets pervers sur la qualité des pratiques de soins.

Le modèle théorique sur lequel repose la tarification à l'activité (T2A) est le modèle de Shleifer (1985). En théorie, la règle de paiement est énoncée ex ante et les forfaits sont calculés ex post. Ex ante, le régulateur annonce aux hôpitaux qu'ils recevront un forfait par GHM, dont le montant n'est pas encore établi mais qui correspondra au coût moyen. L'originalité de ce modèle est de proposer un forfait reposant sur une moyenne de coût tronquée. En théorie, le forfait est calculé comme la moyenne des coûts à l'exclusion de l'hôpital recevant le forfait. Ce modèle repose sur des hypothèses très contraignantes : l'homogénéité des hôpitaux et l'homogénéité des patients. Le seul paramètre de coût qui varie est le niveau d'effort de réduction de coût. Face à tout paiement forfaitaire, le comportement rationnel est de chercher à capter la rente entre le forfait et le coût effectif. Ici, il s'agira de fournir l'effort maximal pour un coût minimal. Ce modèle a pour objectif de pallier l'asymétrie d'information d'un régulateur qui n'observe pas les coûts réels des établissements.

Dans les faits, il n'y a pas parfaite homogénéité des hôpitaux et des patients. Les déterminants de la variation des coûts peuvent être multiples. Ainsi, pour la mise en application, les forfaits sont calculés de façons variables suivants les pays (Annexe D). Ce qui importe est que le forfait reçu pour un séjour soit exogène au coût de l'établissement. Les établissements sont donc incités à réduire leurs coûts et à améliorer l'utilisation de leurs ressources pour une meilleure productivité si les tarifs sont fixés au niveau des établissements les plus efficients. Il apparaît que la subtilité du paiement réside dans le calcul des forfaits.

Une telle tarification ne permet aucun contrôle sur le volume des soins. En France, un mécanisme d'enveloppe fermée accompagne la logique de tarification par GHM : le budget total est défini ex-ante par la loi de financement de la sécurité sociale (LFSS). Ainsi après calcul de la « moyenne des coûts » par $\mathrm{GHM}^{3}$, une règle de 3 est appliquée afin de mettre en adéquation le volume des dépenses prévisionnelles et le budget total défini par la LFSS. Ainsi, le montant du forfait par séjour dépend de l'activité globale réalisée par l'ensemble des établissements de santé sur le territoire. Le taux d'augmentation des enveloppes sous la T2A étant très faible, la tarification de forfait par GHM complétée par le mécanisme d'enveloppe fermée conduit à une forte incitation de réduction du coût par GHM pour l'ensemble des établissements. Se traduit-elle par une forte homogénéité des coûts intra-GHM ?

\footnotetext{
${ }^{3}$ Le calcul de la « moyenne des coûts » par l'ATIH est plus complexe qu'une simple moyenne des coûts. Ils prennent en compte des aspects géographiques et de politiques d'organisation des soins.
} 
Le modèle de Shleifer ne se prononce pas sur la qualité mais il existe une large littérature sur les limites de ce modèle et sur les effets pervers de la T2A sur la qualité lorsqu'elle est endogène (Ellis et McGuire, 1986, 1990 ; Pope, 1989, Ma, 1994 ; Chalkley et Malcomson, 1998, 2000 ; Mougeot et Naegelen, 2005 ; Brekke et Sogard, 2007). Ces effets pervers sont d'autant plus importants que pour un forfait donné, la variabilité des coûts est grande. Cette étude contribue à la littérature sur les liens entre incitations financières et offreurs de soins. Les analyses proposées ne traitent pas directement de la question de la concurrence ni de la qualité des soins. L'objectif de ce papier vise à étudier la variabilité des coûts hospitaliers pour des séjours comparables en pathologies et en procédures (Groupes homogènes de séjours GHS $)^{4}$ Nous analysons ici i) quelle part de variance des facteurs observables n'est pas prise en compte par les GHM ; ii) En quoi les caractéristiques observables durant le séjour du patient peuvent expliquer les écarts permanents de coûts moyens entre les établissements.

Les données utilisées sont par séjour hospitalier (McClellan, 1997; Kessler and McClellan, 2002; Dormont and Milcent, 2004; Olsen and Street, 2008; Laudicella et al., 2010; Dormont and Milcent, 2005; Bradford et al., 2001). Nous utilisons la base de données utilisée pour la fixation des tarifs de Groupe Homogène de Malades (GHM) sur les années 2006 à 2011. Tous les séjours sont codés dans la version 11 des GHM et portent sur des patients atteints d'infarctus du myocarde. Cette base de données contient des informations précises sur le niveau de sévérité de l'état de santé. Ces données sont collectées en routine. Par ailleurs, cette base fournit également le coût par séjour mesuré suivant la même comptabilité analytique quel que soit l'établissement.

La variabilité des coûts est étudiée en deux temps. Dans un premier temps, nous effectuons une analyse de la variance. Puis, nous expliquons le coût du séjour à l'aide d'un modèle à effets fixes hôpitaux, prenant en compte la tendance temporelle.

La tarification à l'activité a pour objectif d'éliminer la variance due aux écarts de coûts entre des séjours comparables en pathologies et en procédures (un même GHM). Sur la période 2006-2011, cette variance représente un tiers de la variance totale. La baisse, attendue avec à la fois l'introduction progressive de la T2A et le raffinement de la classification, apparaît, sans toutefois parvenir à une homogénéisation des coûts. Ainsi, une part non négligeable d'hétérogénéité des coûts subsiste. Ceci laisse place à de possibles effets néfastes sur la qualité des soins fournis. Toutefois, cette hétérogénéité des coûts intra-GHM décroît au cours de la période 2006-2011. Par ailleurs, ce résultat présente des disparités suivant le statut de l'hôpital: les établissements privés ont une dispersion des coûts plus homogènes: ils présentent moins de différences de coûts en termes d'efficacité, de qualité, ou d'hétérogénéité des patients admis. Nous montrons également que les caractéristiques observables du patient durant son séjour expliquent davantage le coût du séjour que ce qui est obtenu par un modèle expliqué par la classification des GHM. Toutefois, les GHM capturent également une part d'information non contenue par ces variables.

Nous analysons également l'importance des spécificités permanentes des établissements comme part explicative de la variance de coût du séjour. Cette part représente $1 / 5$ de la variance du coût et diminue sur la période 2006-2011. Elle ne semble pas être due à des comportements stratégiques en termes de durée de séjour.

Malgré les limites d'une comparaison avec une étude antérieure sur la France, quelques signaux de changement semblent apparaître. Les résultats tendent à montrer que la part

\footnotetext{
${ }^{4}$ Dans la suite du texte, nous confondrons de façon abusive les termes GHM et les GHS. Les GHS sont une déclinaison plus fine des GHM. Ils prennent en compte certains cas spécifiques nécessitant le découpage des GHM en sous-catégories. Le nombre de GHS est très légèrement supérieur au nombre de GHM.
} 
expliquée par la classification des GHM s'est réduite. Par ailleurs, les caractéristiques observables du patient semblent mieux prises en compte par la classification actuelle.

La partie 1 présente le tissu hospitalier français et les évolutions de tarification. La partie 2 commente la variabilité des coûts hospitaliers. La partie 3 expose les résultats du modèle de coût. La conclusion et la discussion sont présentées en partie 4 .

\section{Hôpitaux français et tarification}

Le tissu hospitalier français se compose de trois types d'établissements de santé : les établissements publics, les établissements privés à but lucratif et les hôpitaux privés à but non lucratif appelés établissements de santé privés d'intérêt collectif (ESPIC).

\subsection{Vers une mise en place d'une tarification basée sur les GHM}

Jusqu'en 2005, les hôpitaux privés à but lucratif facturaient directement à l'assurance maladie des forfaits de prestations (rémunération de la structure) et des actes (correspondant aux rémunérations des professionnels de santé libéraux). Les forfaits de prestations étaient encadrés par l'Objectif Quantifié National (OQN) assurant une régulation de type «prix/volume». Les forfaits des prestations variaient d'une région à l'autre, en fonction des négociations entre les agences régionales d'hospitalisation $(\mathrm{ARH})$ et fédérations régionales.

Les hôpitaux publics et les établissements de santé privés d'intérêt collectif (ESPIC), partageaient le même mode de financement ainsi que les mêmes missions de service public. Ces hôpitaux étaient financés sous forme de budget global à partir de 1983. La mise en place de ce budget avait pour but de maîtriser les dépenses. Le financement accordé aux hôpitaux était décidé sur la base des dépenses observées l'année antérieure avec une prise en compte d'un coefficient d'inflation des coûts hospitaliers. Le mécanisme de fixation du budget a fait peser une contrainte hétérogène sur les établissements de santé. Pour les établissements qui avaient une maitrise de leurs dépenses, le budget global est rapidement devenu une contrainte lourde pour leur activité. De façon symétrique, la contrainte était lâche pour les établissements de santé qui n'opéraient que peu de contrôle des dépenses lors de la mise en place de ce mécanisme. Au cours du temps, avec la nécessité de réduction des dépenses de santé, la contrainte financière s'est serrée. La caractérisation première de la politique de budget global telle que mise en place en France est qu'elle a été réalisée sans information sur l'activité réelle des établissements de santé. Il n'y avait pas adéquation entre " contrainte financière » et « activité hospitalière ».

La forte volonté de rapprocher le financement des établissements de santé à leur activité réelle a conduit à l'adoption de la tarification à l'activité (T2A) en 2004. Aujourd'hui, les deux secteurs public et privé sont soumis au même mode de tarification ${ }^{5}$. La T2A est basée sur la classification de chaque séjour en un GHM unique.

Pour le secteur privé, la réforme a été adoptée le ler Mars 2005. Auparavant, ces établissements de santé étaient déjà largement dans un système de marché concurrentiel. Dès

\footnotetext{
5 Il serait abusif de dire que les deux secteurs sont soumis à la même tarification puisqu'ils ne partagent pas la même grille de tarifs : pour un même GHM, le forfait associé est différent suivant le secteur public ou privé.
} 
le passage à la T2A, les hôpitaux privés à but lucratif ont été remboursés à $100 \%$ par forfait en fonction du GHM associé au séjour.

Pour le secteur public, la mise en place de la T2A a été graduelle. Il s'agissait de permettre un ajustement des pratiques de gestion et d'organisation des établissements de santé. En effet, la tarification basée sur leur activité, introduit la concurrence entre établissements. De 2004 à 2007, les établissements ont bénéficié d'une tarification mixte :

- Un pourcentage de budget global (BG). Durant cette période, le montant du BG était établi tel qu'il aurait été établi sans la mise en place de la tarification à l'activité (T2A). Cette partie de la tarification couvrait l'ensemble des séjours réalisés dans l'hôpital sans prise en compte explicite du volume des soins produits ;

- Un pourcentage du forfait par séjour. Le montant de ce forfait est défini en fonction du GHM associé au séjour. Cette partie du financement étant par unité, le volume des soins produits par l'hôpital est pris en compte.

L'évolution du passage à la T2A a été très progressive en début de période pour s'accélérer entre 2007 et 2008 et, atteindre $100 \%: 10 \%$ en $2004,25 \%$ en $2005,35 \%$ en $2006,50 \%$ en 2007 et $100 \%$ en 2008 .

La période de tarification par budget global était une période de grande rationalisation à l'aveugle au vu de la période antérieure (jusqu'en 1983). Sous la T2A, si la tarification est accompagnée d'un système d'information sur l'activité des établissements permettant un mode de financement éclairé, la contrainte financière est encore plus forte.

\subsection{Forte augmentation du nombre de groupe de GHM}

Selon les pays ayant mis en place une tarification par GHM, le nombre de groupe varie très fortement : entre 400 et plus de 3000 groupes. La classification des GHM doit arbitrer entre suffisamment de séjours hospitaliers par groupe pour que le forfait soit représentatif du coût d'une activité et non un forfait remboursant une procédure en particulier; et, des groupes suffisamment fins pour permettre une homogénéité de coût de façon à ne pas introduire d'incitation à la sélection de patients ou de certaines procédures au détriment d'autres.

Depuis la création des GHM, différentes classifications se sont succédées. En 2009, le nombre de groupes est presque multiplié par 3 (près de $2200 \mathrm{GHM}$ ). Ce changement s'accompagne d'un changement de logique dans la construction des GHM. Les GHM sont désormais pensés en "poupées russes ». Ils sont codés sur 6 caractères dont le sixième définit le niveau de sévérité. Les groupes ainsi définis, ont un tronc commun correspondant aux 5 premiers caractères appelés «racines». Il existe environ 600 racines. Pour une explication plus détaillée, se rapporter à l'Annexe $B$.

Le système de classification des GHM joue un rôle central pour l'équité des paiements entre les hôpitaux. Une large littérature étudie les effets d'un paiement par GHM sur la sélection des patients ou discrimination par niveaux de sévérité ou comorbidités. Hafsteinsdottir et Siciliani (2008) discutent des effets d'une classification fine des GHM versus une classification plus grossière. Ils montrent qu'avec une classification plus fine, les acteurs de soins ont une plus grande incitation à fournir des actes chirurgicaux. A l'inverse, une classification plus sommaire désinciterait à la réalisation de procédures chirurgicales. Aux Etats-Unis, les séjours de patients traités par certaines procédures chirurgicales sont associés à des GHM spécifiques (McClellan, 1997 ; Gilman, 2000). 


\section{Coûts hospitaliers et variabilité}

\subsection{Données}

Les données mobilisées sont celles issues de la base de l'Etude Nationale de Coût à méthodologie Commune (ENCC) pour des patients atteint d'infarctus aigu du myocarde sur la période 2006-2011.

L'ENCC contient d'une part des informations médicales précises sur les diagnostics, traitements et procédures réalisés durant le séjour hospitalier. Il s'agit d'informations recueillies en routine.

L'ENCC contient d'autre part des données de coûts relatives à la prise en charge des patients hospitalisés. Les établissements publics comme privés peuvent participer à cette base lorsque leur comptabilité analytique est jugée fiable par l'Agence Technique de l'Information sur l'Hospitalisation (ATIH). La participation à l'ENCC est basée sur le volontariat. La motivation principale pour un établissement de participer à cette étude est le besoin d'objectiver sa spécificité de son activité médicale en termes de coûts.

Les données médico-économiques utilisées - les coûts par GHM - constituent le produit fini de l'ENCC. Ils permettent à l'ATIH d'élaborer les deux échelles de coûts différentes, l'une pour le secteur public, l'autre pour le secteur privé. Lors de l'étape technique d'élaboration des tarifs des $\mathrm{GHM}^{6}$, les coûts moyens de prise en charge supportés par les établissements sont réajustés en fonction de divers objectifs de la tutelle. La corrélation tarifs-coût moyen varie donc en fonction du GHM considéré. La mise en évidence d'une très forte variabilité des coûts au sein d'un groupe homogène de malades (GHM) peut conduire à faire évoluer la classification des GHM vers un nouveau GHM et de ce fait, à introduire un forfait supplémentaire pour ce type de séjour.

Sur la base des différents diagnostics et actes pratiqués sur le patient durant son séjour et à l'aide d'une arborescence, les séjours sont codés dans la version 11 des GHM sur l'ensemble de la période. L'information commune de la version des GHM permet une comparaison temporelle pour les analyses de variabilité.

Les données utilisées ici sont des données pour l'infarctus aigu du myocarde (IAM), sélectionnées à partir des diagnostics principaux de la classification internationale des maladies, version 10 (CIM-10) et codées dans les 13 GHM version 11 pour IAM. L'échantillon contient 52732 observations sur les 5 années. Une présentation de la fréquence et des coûts associés à ces GHM est donnée Tableau 3.

Les données pour crise cardiaque comportent un certain nombre d'avantages. D'une part, le volume des admissions pour ce type de pathologie est relativement important. Les maladies ischémiques représentent l'une des causes majeures de décès en France. D'autre part, les infrastructures utilisées pour soigner les patients sont proches d'un hôpital à un autre. Par ailleurs, d'après Gaynor (2006), la mortalité pour IAM est un bon marqueur de la qualité des soins au sein de l'établissement de santé. Ensuite, il est usuellement considéré que les patients

\footnotetext{
${ }^{6}$ Un forfait est en fait associé à chaque groupe homogène de séjour (GHS) qui correspond à un léger redécoupage des GHM.
} 
atteints d'infarctus du myocarde sont admis dans l'hôpital le plus proche ce qui laisse peu de place au problème de sélection des patients (Gaynor et al., 2013). La pathologie « infarctus aigu du myocarde » est donc le sujet d'un grand nombre d'études sur l'hétérogénéité des indicateurs de qualité et/ou la variabilité des coûts des établissements hospitaliers (Kessler and McClellan, 2000 ; Kessler and Geppert, 2005 ; Gowrisakaran and Town, 2003 ; Bloom et al., 2010 ; Cooper et al., 2011 ; Gaynor et al., 2013).

L'hétérogénéité des patients est contrôlée à partir non seulement de l'âge, du sexe mais aussi de l'ensemble des diagnostics et des comorbidités relevés durant le séjour ainsi que les actes et procédures réalisés durant le séjour. Malgré la précision de ces informations et le choix de la pathologie favorisant l'admission à l'hôpital de proximité, la possibilité de biais de sélection peut être discutée. McClellan and Staiger (1999) montrent que les comorbidités et diagnostics considérés ici suffisent à capturer l'hétérogénéité des patients.

La structure des données est assez complexe. Il s'agit de données stratifiées à trois dimensions: séjour-hôpital-année. i) Les données sont non cylindrées dans plusieurs dimensions : le nombre de séjours varie d'une part en fonction de l'hôpital considéré, d'autre part en fonction de l'année considérée. ii) Les hôpitaux ne sont pas présents sur l'ensemble de la période étudiée. La base ENCC étant basée sur le volontariat, les hôpitaux entrent et sortent de la base en fonction des années. Le Tableau 1 donne la répartition des hôpitaux par année. En 2008, les établissements privés ont largement boycotté leur participation à la base ENCC après un appel de la fédération des établissements privés (FHP). Il s'agissait d'un mouvement de contestation du recul de la convergence tarifaire. Les établissements privés souhaitaient que le forfait par GHM soit le même quel que soit le type d'établissement. De même une partie des Centres Hospitaliers Régionaux (CHR) n'ont plus participé à la base ENCC les deux dernières années de mise en place de la $\mathrm{T} 2 \mathrm{~A}$ pour ensuite participer pleinement à l'enquête.

Des critiques peuvent être émises sur la représentativité de la base de coût. Toutefois, aujourd'hui elle fournit les uniques données de coût menant à l'élaboration des forfaits par GHS. Elle est le socle de la T2A. Ces forfaits sont ensuite appliqués pour l'ensemble des hôpitaux français, à la base de la T2A.

Il peut également se poser la question du cylindrage de l'échantillon. D'une part, cylindrer l'échantillon entrainerait la perte d'un grand nombre d'hôpitaux et donc d'observations; D'autre part, cette base est la représentation qu'a la tutelle de l'ensemble des établissements en France pour le calcul des forfaits. Si les hôpitaux participent volontairement à l'Etude Nationale de Coût à méthodologie Commune (ENCC), la tutelle se réserve le droit de refuser la participation de certains établissements ou de ne pas les inclure in fine afin d'assurer une représentativité adéquate des hôpitaux participants vis à vis de l'ensemble des hôpitaux français autant par leur taille que par leur statut.

\subsection{Caractéristiques des séjours}

Le Tableau 1 présente les statistiques générales de la base de données. Le nombre de séjours ainsi que le nombre d'hôpitaux sont en augmentation sur la période.

\footnotetext{
7 Ce point a été confirmé par la responsable du département ENCC de la tutelle en charge des bases de données du Programme Médicalisé des Systèmes d'Information (PMSI).
} 
Les caractéristiques des séjours sont données Tableau 2. Globalement, les patients sont des hommes âgés entre 65 et 89 ans. La durée moyenne de séjour est de 6 jours et demi et la médiane est de 5 jours. Les patients proviennent massivement de leur domicile (plus de 4/5 des patients) et deux-tiers d'entre eux retournent à leur domicile.

Les statistiques présentées Tableau 3 donnent la répartition des séjours entre les différents GHM. Plus d'un tiers des séjours sont codés par le GHM 05K01. Les séjours se répartissent dans 4 racines de GHM subdivisées en fonction du niveau de gravité. Les GHM chirurgicaux (lettre « $\mathrm{K} »$ en troisième position) sont davantage représentés $(65 \%)$ que les GHM médicaux (lettre « $\mathrm{M}$ » en troisième position). Les coûts moyens par GHM révèlent que les séjours classés dans les GHM chirurgicaux sont plus coûteux. Toutefois, les vraies différences de coûts portent principalement sur les niveaux de sévérité. Ainsi pour une même racine de GHM chirurgicaux, le coût varie entre $5600 €$ pour un niveau de sévérité faible à $23800 €$ pour un niveau de sévérité très élevé.

Les actes techniques sont très fréquemment utilisés. Un stent est utilisé après un cathétérisme et une angioplastie. Deux tiers des patients sont traités par un stent $(64,41 \%)$. Une angioplastie est réalisée après un cathétérisme $(4,54 \%)$. Les cathétérismes seuls concernent $26,4 \%$ des patients.

\subsection{Variabilité des cô̂ts des séjours}

Pour une année donnée, en raisonnant tous hôpitaux confondus, nous pouvons calculer un coût moyen par GHM, noté $\mathrm{C}_{\mathrm{g}}$ avec $\mathrm{g}$, le GHM (g=GHM 05K051, 05K052, ..., 05M164). La variance du coût des séjours se décompose de la façon suivante :

$$
V\left(C_{g, i}\right)=V\left(C_{g}\right)+V\left(C_{g, i}-C_{g}\right)
$$

L'indice i désigne le séjour. La variance totale des coûts, notée $V\left(C_{g, i}\right)$ est égale à la somme de

- La variance inter-GHM $\mathrm{V}\left(\mathrm{C}_{\mathrm{g}}\right.$. $)$ : due aux écarts de coûts moyens entre les GHM.

- La variance intra-GHM, $\mathrm{V}\left(\mathrm{C}_{\mathrm{g}, \mathrm{i}}-\mathrm{C}_{\mathrm{g}}.\right)$ : due aux écarts de coûts entre les séjours d'un même GHM.

La tarification hospitalière est basée sur un forfait par GHM : un hôpital qui a la même distribution de séjour/GHM qu'un autre hôpital a le même budget de fonctionnement pour son activité. La tarification suppose que d'une part les patients sont parfaitement homogènes; d'autre part, les hôpitaux soignent les patients de façon identique. Nous nous proposons de vérifier cette hypothèse grâce à un indicateur. Il s'agit de calculer la part de la variance des coûts due aux différences de coûts moyens entre les $\mathrm{GHM}\left(\mathrm{V}\left(\mathrm{C}_{\mathrm{g}}.\right) / \mathrm{V}\left(\mathrm{C}_{\mathrm{g}, \mathrm{i}}\right)\right)$.

Si pour un GHM donné, d'une part les patients étaient parfaitement homogènes ; d'autre part, les hôpitaux soignaient de façon identique les patients, alors la variance inter-GHM serait exactement égale à la variance totale. La variance intra-GHM serait nulle $\left(V\left(C_{g, i}-C_{g}\right)=0\right)$.

Trouver une valeur proche de 1 signifie que l'hypothèse est vérifiée. Moins l'hypothèse est vérifiée, plus l'indicateur est proche de zéro. Une valeur faible indique une forte hétérogénéité des coûts pour un même GHM.

Le même type de raisonnement peut être appliqué pour étudier la part de la variance des coûts attribuable aux différences de coûts moyens entre les hôpitaux. En raisonnant pour un GHM 
donné et une année donnée, nous pouvons calculer un coût moyen par hôpital, noté $\mathrm{C}_{\mathrm{h}} \mathrm{avec} \mathrm{h}$, l'hôpital $(\mathrm{h}=1, \ldots, 328)$. La variance du coût des séjours se décompose de la façon suivante :

$$
V\left(C_{h, i}\right)=V\left(C_{h} .\right)+V\left(C_{h, i}-C_{h} .\right)
$$

La variance totale des coûts, notée $\mathrm{V}\left(\mathrm{C}_{\mathrm{h}, \mathrm{i}}\right)$ est égale à la somme de

- La variance inter-hôpitaux $\mathrm{V}\left(\mathrm{C}_{\mathrm{h}}\right.$.) : due aux écarts de coûts moyens entre les hôpitaux.

- La variance intra-hôpital $\mathrm{V}\left(\mathrm{C}_{\mathrm{h}, \mathrm{i}}-\mathrm{C}_{\mathrm{h}}\right.$.) : due aux écarts de coûts entre les séjours d'un même hôpital.

La part de la variance des coûts attribuable aux différences de coût moyen de l'hôpital est :

$$
V\left(C_{h} .\right) / V\left(C_{h, i}\right)
$$

Elle correspond à la variance des coûts qui serait due à des caractéristiques permanentes des hôpitaux. Ces caractéristiques peuvent correspondre aux différences d'efficience, de qualité ou toutes autres différences à condition qu'elles soient permanentes c'est à dire qu'elles touchent l'ensemble des séjours admis dans l'hôpital $h$.

L'hypothèse d'homogénéité des hôpitaux peut être testée par la part de la variance interhôpitaux par GHM. La variance des coûts peut d'autant plus être attribuée à l'hétérogénéité des hôpitaux que cette part est proche de 1 .

\subsection{Les résultats de la variabilité des coûts hospitaliers}

Nous montrons que $68 \%$ de la variance totale des coûts est due à la variance inter-GHM des coûts (Tableau 4). Ainsi, un tiers de la variance totale des coûts est due à la dispersion des coûts autour des valeurs moyennes. Dit autrement, malgré la tarification à l'activité qui a pour objectif d'éliminer la variance due aux écarts de coûts entre les séjours d'un même GHM, cette variance représente un tiers de la variance totale.

Notre période d'observation couvre la mise en place de la tarification à l'activité (T2A). En 2006 , la T2A était déjà introduite à $100 \%$ pour les établissements privés à but lucratif. En revanche, les hôpitaux du service public étaient payés à $35 \%$ par T2A et à $65 \%$ par budget global. La T2A a été appliquée à $100 \%$ dans les deux secteurs en 2008. La T2A est une réforme pro-compétition. On s'attend donc à ce que la mise en place complète de la T2A conduise à diminuer la variance des coûts pour un GHM donné. Dit autrement, la T2A devrait conduire à des coûts peu dispersés pour un GHM donné. Les résultats font apparaître une baisse : les coûts sont moins dispersés au cours du temps (Tableau 4). Ces résultats suggèrent que la T2A et le raffinement de la classification conduisent à estomper l'hétérogénéité des coûts par GHM, au cours du temps.

Nous montrons également que les coûts par GHM des séjours dans des hôpitaux publics (100$67=33 \%)$ sont plus dispersés que ceux des hôpitaux privés $(100-78=22 \%)$. Ce résultat est également obtenu lorsque l'on s'intéresse à la variance intra-GHM des coûts par année et type d'hôpital (Tableau 4). Ainsi les établissements privés à but lucratif, pour des séjours comparables en pathologies et en procédures, ont une dispersion des coûts plus homogène : ils présentent moins de différences de coûts en termes d'efficacité, de qualité, ou d'hétérogénéité des patients admis.

Par ailleurs, nous montrons que la variance des coûts due à des différences de coûts moyens entre les hôpitaux est de $19 \%$. Les différences de coûts pour un même séjour entre deux hôpitaux peuvent être expliquées par des différences de qualité ou des différences d'efficacité des établissements ou encore des différences de caractéristique de patient. Ne pas rembourser ces écarts de coûts semble légitime et même souhaitable pour des différences d'efficacité 
mais semble problématique pour des différences de qualité ou d'hétérogénéité des patients (Tableau 5).

Ainsi, il est possible que cette part importante soit due à des différences de caractéristiques des patients pris en charge. Imaginons que certains hôpitaux ne traitent que des patients à pathologie sévère et que d'autres hôpitaux ne traitent exclusivement que des patients à pathologie bénigne, le résultat serait une variance inter-hôpitaux importante. Pour examiner ce cas, nous avons calculé la variance inter-hôpitaux par GHM. Les résultats montrent également une forte variance inter-hôpitaux. Pour chaque GHM considéré, certains hôpitaux ont systématiquement des coûts plus élevés quel que soit le niveau de sévérité du patient et le type de soins donnés au patient, médical ou chirurgical. L'explication est alors i) des différences de caractéristiques médicales des patients entre les établissements, suggérant largement une sélection des patients ; ii) des modes de prise en charge systématiquement plus coûteux pour certains établissements.

On peut s'attendre à ce que la variance des coûts intra-établissement baisse avec l'introduction progressive de la T2A et le raffinement de la classification des GHM. Les Graphiques 1 présentent les évolutions de la variance intra-hôpital pour 3 GHM. Il s'agit des trois GHM les plus codés de l'échantillon. Malgré des fluctuations sur la période, nous observons une tendance à la baisse.

Les comparaisons d'analyse de la variance dans le temps ne posent pas de problème lorsque l'échantillon est cylindré sur la période d'observation. Ici, comme le montre le tableau 1, le nombre d'hôpitaux fluctue au cours du temps. Notre analyse repose donc sur une représentativité constante des hôpitaux de la base de coût avec l'ensemble des hôpitaux présents sur le territoire français. Si cette hypothèse peut-être discutée, il s'agit de celle faite par la tutelle pour l'utilisation de la base ENCC à la fixation des tarifs.

Nous avons mis en évidence l'existence d'une variance inter-hôpitaux. Toutefois, nous ne savons pas comment se constitue cette variance. Est-elle due à des différences de niveau de qualité, des différences d'efficience ou encore à des différences de composition des patients ? Mécaniquement, la sortie de l'ENCC d'un établissement extrême, toutes choses égales par ailleurs, réduit la variance observée intra-établissement ${ }^{8}$. Travailler GHM par GHM réduit la taille de l'échantillon rendant les résultats plus sensibles à toute modification de l'échantillon. Il est souhaitable de ne pas rembourser des différences liées à des inefficiences alors qu'il n'est pas pensable de pénaliser les hôpitaux prenant en charge des patients plus lourds financièrement pour un GHM donné. Il importe donc de donner une évaluation plus précise de la part de la variance des coûts attribuable aux écarts permanents de coûts moyens entre les hôpitaux d'une part et à l'hétérogénéité des patients non prise en compte par les GHM d'autre part. Pour cela, en partie suivante, nous évaluons la part de variance expliquée par des facteurs observables avant et après la prise en compte des GHM comme variable explicative. Nous évaluons également la part de la variance expliquée par les effets fixes hôpitaux correspondant aux écarts permanents de coûts entre les hôpitaux, après prise en compte des facteurs observables.

\footnotetext{
${ }^{8}$ Ceci est également vrai pour la variance intra-GHM
} 


\section{Coûts hospitaliers : Variance expliquée par les GHM versus Variance expliquée par des facteurs observables}

Nous raisonnons maintenant en prenant un patient de référence. Ses caractéristiques dépendent du modèle considéré. Ici, l'objectif n'est pas d'expliquer les coûts per se mais de déterminer la part de la variance expliquée par les GHM et de les mettre en regard à la part de variance expliquée par les variables observables du modèle. La question posée ici est : est-ce que la classification capture suffisamment l'hétérogénéité des coûts pour éviter les effets néfastes de sélection des patients ou de diminution du niveau de qualité offert que peut engendrer une tarification par forfait. La seconde question porte sur les déterminants des écarts permanents de coûts moyens entre les établissements hospitaliers. La méthodologie appliquée ici se réfère au papier de Dormont et Milcent (2000). Cette stratégie de modélisation est également proche de celle développée dans le projet EuroDRG présentée dans une série d'articles dans la revue Health Economics en 2012.

\subsection{Le modèle}

Les modèles estimés sont de la forme :

$$
C_{i, g, h, t}=\theta D d S_{i, g, h, t}+\delta A C T E_{i, g, h, t}+\gamma X_{i, g, h, t}+\beta_{h}+\alpha_{g}+\kappa T_{t}+u_{i, g, h, t}
$$

Avec,

- $\quad C_{i, g, h, t}$ le coût du séjour $i$ associé au GHM $g$ réalisé dans l'hôpital $h$, l'année $t$

- $D d S_{i, g, h, t}$ la durée du séjour $i$ associée au GHM g réalisé dans l'hôpital $h$, l'année $t$

- $A C T E_{i, g, h, t}$ les actes et procédures réalisés lors du séjour $i$ associé au GHM $g$ réalisé dans l'hôpital $h$, l'année $t$

○ Cathétérisme

- Angioplastie

○ Stent

- $\quad X_{i, g, h, t}$ les caractéristiques du patient admis pour le séjour $i$ associé au GHM $g$ réalisé dans l'hôpital $h$, l'année $t$

○ Age, sexe

- Diagnostics

- Comorbidités

- $\quad \beta_{h}$ les variables indicatrices des hôpitaux $h$

- $\alpha_{g}$ les variables indicatrices des GHM $g$

- $T_{t}$ la tendance temporelle

- $u_{i, g, h, t}$ le terme d'erreur

Les modèles sont estimés à la fois avec le coût de séjour et le logarithme des coûts. Dans la littérature empirique, les coûts sont souvent modélisés en prenant le logarithme du coût. D'abord, ici, les tests de Skewness et Kurtosis ne permettent pas de trancher entre un modèle linéaire et un modèle log-linéaire. Ensuite, ce qui pourrait dicter un comportement stratégique de la part de l'hôpital est le niveau du coût du séjour et non son logarithme. Enfin, avoir les deux estimations permet de voir si des valeurs élevées de variance des coûts sont liées à quelques séjours particulièrement coûteux, le modèle log-linéaire " gommant » l'effet de ces valeurs élevées.

La prise en compte de la durée de séjour comme variable explicative du coût du séjour doit être discutée. Cette variable peut être non exogène. L'hôpital peut réduire intentionnellement la durée du séjour afin de réduire le coût du séjour. Si l'hôpital adopte le même comportement 
à tous les séjours, le comportement stratégique de l'hôpital jouera sur la durée moyenne des séjours. Un modèle pertinent serait celui qui inclurait les effets fixes hôpitaux. La durée apparaîtrait alors comme un déterminant des écarts permanents de coûts moyens entre les établissements hospitaliers. En revanche, si les hôpitaux adaptent la durée en fonction du séjour et de leur volonté à réduire le coût, les modèles proposés ici contrôlant par la durée, seront biaisés. Ceci est également vrai pour le modèle à effets fixes hôpitaux.

\subsection{Le coût d'un séjour pour crise cardiaque}

Les résultats de l'équation (1) présentée ci-dessus sont donnés Tableau 6. Tous hôpitaux confondus et toutes années confondues, le coût moyen d'un séjour pour l'individu de référence est $2400 €$. Ce coût moyen correspond aux caractéristiques de l'individu de référence : un homme âgé entre 41 et 50 ans, venant de son domicile et retournant à son domicile à l'issu du séjour, n'ayant aucun des diagnostics secondaires et comorbidités pris en compte dans le modèle, n'ayant subi ni cathétérisme, ni angioplastie, ni stent et, admis pour un séjour associé au GHM 05K051 (endoprothèses vasculaires et Infarctus Aigu du Myocarde). Pour ce même individu de référence, nous avons calculé la valeur du coût moyen en fonction de l'hôpital d'admission : $3200 €$ en étant admis dans un hôpital public ; $2000 €$ pour une admission dans un hôpital à but non lucratif ${ }^{9}$ et $1300 €$ pour une admission dans un établissement privé à but lucratif. Le forfait correspondant à ce GHM est environ de $2150 €$ pour les établissements privés et de $2800 €$ pour les établissements publics.

Les individus âgés coûtent moins chers que les individus jeunes. Il n'y a pas de différence significative de coût moyen suivant le genre.

Les patients provenant d'un autre hôpital ont un coût de séjour moins élevé (mais non significatif). En revanche, les patients transférés vers un autre hôpital ont un coût de séjour significativement plus élevé $(+200 €)$. Le décès augmente significativement le coût du séjour $(+1000 €)$.

A niveau de sévérité identique ou légèrement supérieur, les séjours codés par des GHM, autres que celui de référence, sont moins chers après contrôle par les actes et les diagnostics secondaires et comorbidités, au niveau de significativité de $0.1 \%$. Le GHM de référence est le plus important en volume de séjours.

Les coefficients obtenus pour les variables indicatrices des actes donnent, toutes choses égales par ailleurs, le surcoût qui leur est associé. La hiérarchie des coûts moyens entre ceux-ci est respectée. Le stent est sur la liste restrictive des actes dit « en-sus ». Cette procédure est donc à considérer différemment puisque l'hôpital reçoit un paiement additionnel pour cet acte.

\subsection{Quelle part de variance des facteurs observables n'est pas prise en compte par les GHM?}

Le graphique 2 présente l'évolution temporelle. Si la part de variance expliquée par les GHM reste insuffisante, elle augmente dans le temps et particulièrement après 2009 avec le raffinement de la classification des GHM.

\footnotetext{
${ }^{9}$ Les hôpitaux à but non lucratif sont appelés Etablissements de santé à but non lucratif qualifiés d'établissements de santé privés d'intérêt collectif, donnant lieu à l'acronyme ESPIC.
} 
Nous évaluons maintenant les apports respectifs des différentes catégories de variables. Nous présentons le $\mathrm{R}^{2}$ associé à chacun des modèles. Les modèles sont présentés de façon incrémentale. A chaque ajout de catégorie de variables, on obtient le surcroît de variance expliquée par ces variables. Le modèle dit de «base » est le modèle expliqué uniquement par les indicatrices de GHM et la tendance temporelle. Le modèle dit " complet » est le modèle qui a été présenté Equation (1). Ce travail nous permet d'identifier l'importance des caractéristiques des patients et des hôpitaux dans la variance expliquée (Tableau 7).

En préambule, nous considérons le modèle où le coût des séjours est expliqué par les sévérités des GHM. Les niveaux de sévérité à eux seuls expliquent 13,3\% de la variance du coût total. Nous présentons également le modèle où le coût des séjours est expliqué par les racines des GHM. Les racines expliquent $6 \%$ de la variance du coût total (Tableau 7). ${ }^{10}$

A partir du groupe de modèles noté A, la part de variance expliquée lorsque le modèle (A1) ne comporte que les indicatrices de GHM et la tendance est de 30,2\%. En ajoutant les caractéristiques des individus (le modèle A2), la part de variance expliquée est alors de 35,45\%. Les GHM ne captent pas l'ensemble de l'hétérogénéité des séjours. Contrôler par les effets fixes hôpitaux augmente la part explicative de $+4 \%$. Nous ajoutons ensuite les procédures. La part de variance expliquée augmente de $+17 \%$. Puis nous prenons en compte la durée, $+14 \%{ }^{11} \mathrm{Au}$ total, $71 \%$ de la variance des coûts est expliquée. Après prise en compte de la classification des GHM, l'hétérogénéité observable entre les patients explique encore une part importante de la variance des coûts.

Sans prise en compte des GHM, les caractéristiques individuelles des patients, les diagnostics et les procédures apportent $43 \%$ de la variance des coûts (le modèle F1). L'hétérogénéité observable entre les patients explique à elle seule une part de la variance des coûts plus importante que la classification des GHM.

Nous montrons également que la classification des GHM apporte une part d'information sur la variance des coûts qui n'est pas capturée par les caractéristiques observables des patients : comorbidités, diagnostics, informations démographiques et procédures réalisées durant le séjour. Cette part de variance expliquée est loin d'être négligeable. Avec le modèle loglinéaire qui a l'avantage de «gommer » les coûts élevés, elle atteint 15,7\%.

En conclusion, nous pouvons dire que les GHM ne capturent pas l'ensemble de l'information disponible sur les caractéristiques des patients et les soins fournis dont les procédures. Toutefois, les GHM capturent une part d'information non contenue par ces variables.

\footnotetext{
10 Nous analysons dans le texte le groupe de modèles noté A. Les variables explicatives du coût total de séjour ne sont pas indépendantes entre elles. Ainsi, le surcroît de variance dû à l'ajout d'une nouvelle variable explicative dépend du jeu de variables explicatives déjà présent dans le modèle. En annexe (Tableau A3), nous présentons donc les modèles en jouant sur l'ordre d'ajout des variables explicatives. Pour chaque groupe de modèles, nous partons du modèle le plus simple où le coût total est expliqué par les indicatrices de GHM et la tendance (modèle 1). Le modèle complet est celui présenté équation (1). Pour chacun des groupes de modèle, il correspond à ce qui est appelé Modèle 5. Les résultats présentés ici ne diffèrent pas de ce qui est présenté en Annexe A, Tableau A3.
}

11 Le modèle A5 correspond au modèle dit complet présenté Equation (1). 
Le rôle des spécificités permanentes des hôpitaux se réduit-il lorsque le modèle tient déjà compte de la durée?

Le Tableau 8 présente les modèles donnant des éléments de réponse à cette question.

- Les modèles C contrôlent pour l'ensemble des facteurs observables dans la base ENCC hors durée de séjours. Le modèle $\mathrm{C} 1$ ne prend pas en compte les effets fixes hôpitaux. Le modèle $\mathrm{C} 2$ prend en compte les effets fixes hôpitaux. L'apport de variance par la prise en compte des effets fixes hôpitaux est de $8 \%$.

- Les modèles D expliquent le coût du séjour par l'ensemble des facteurs observables dont la durée de séjour. Le modèle D1 ne prend pas en compte les effets fixes hôpitaux. Le modèle D2 prend en compte les effets fixes hôpitaux. L'apport de variance par la prise en compte des effets fixes hôpitaux est alors de $6 \%$.

- Prendre la variable de coût en logarithme "gomme» l'influence des valeurs extrêmement élevées de coût. La comparaison avec les modèles en log-linéaire aboutit à des résultats encore plus semblables. Il apparaît donc que le surcroît de variance expliqué par des écarts permanents entre les établissements ne permet pas de conclure à des comportements stratégiques des établissements visant à jouer sur les durées des séjours dans le but de diminuer le coût des séjours.

Le surcroit de variance expliqué par les spécificités permanentes entre les établissements hospitaliers est de l'ordre de $5 \%$ à $8 \%$. A supposer que la durée soit exogène, ce surcroît de variance n'est pas dû à des différences de durée de séjour.

Par ailleurs, les spécificités hospitalières expliquent 4 à $7 \%$ de variance du coût total, le statut public, privé ou ESPIC des établissements de santé n'apportent pas d'information supplémentaire (Tableau 8, partie du tableau ayant pour titre «Modèle expliqué par les types d'hôpitaux versus les spécificités permanents des hôpitaux »).

\subsection{Comparaison avec d'autres études}

Dans le cadre du projet EuroDRG, Hakkinen et al (2012) étudient pour l'infarctus aigu du myocarde, la part de variance des coûts expliquée par les GHM. Le modèle est ici log-linéaire. Par ailleurs, ils utilisent un échantillon de séjours pour lequel les patients ayant subi un pontage, procédure entrainant un coût élevé, sont exclus. Pour la France, l'étude est réalisée à partir des données ENCC 2007 et d'une version antérieure des GHM. Ils montrent que 52\% de variance du logarithme des coûts sont expliqués par les $\mathrm{GHM}^{12}$. Ils concluent également que les GHM ne permettent pas de capturer l'ensemble de l'information des facteurs observables des patients et des établissements. Dans leur étude, pour la plupart des pays, le coût est davantage expliqué par les caractéristiques des patients, les diagnostics, les procédures et le mode de prise en charge que par la classification des GHM. Ce résultat est également celui obtenu ici. Ils préconisent une amélioration de la classification des GHM par l'ajout de variables explicatives non seulement sur les niveaux de complications mais aussi les procédures. Mason et al. (2012) pour l'appendicectomie, concluent au même résultat. Hafsteinsdottir et Siciliani (2009) mettent en avant, à l'aide d'un modèle théorique, qu'un

\footnotetext{
12 Pour cette même année 2007, en prenant le logarithme du coût, nous trouvons $45 \%$ de variance des coûts expliqués par les GHM.
} 
raffinement de la classification basée sur les procédures conduit à favoriser les actes techniques (ou chirurgicaux) au détriment de procédures moins invasives.

Dans ce papier, nous avons repris en partie la démarche adoptée par Dormont et Milcent $(2000)^{13}$. Ce papier portait sur 5623 séjours, classés dans 6 GHM et effectués dans des établissements du secteur public pour les années 1994 et 1995. Entre la période 1994-1995 et aujourd'hui, un certain nombre de changements peuvent s'observer (Tableau A1). La durée du séjour s'est fortement réduite depuis les années 1994-1995. Si entre 1994 et 1995, 44\% des patients avaient une durée comprise entre 8 et 14 jours, aujourd'hui ils ne représentent plus que $21 \%$. De même, le taux de décès hospitalier s'est fortement réduit $(4,3 \%)$. Il était de 9,4\% entre 1994 et 1995 et il a été divisé par plus de moitié au cours du temps. Cette évolution est concordante avec ce qui est observé sur les données PMSI pour patients atteints d'infarctus du myocarde sur la période 1998-2011 (Gobillon et Milcent, 2014). Avant de poursuivre cette comparaison, il faut en souligner les limites.

D'abord, les échantillons (le nombre et le profil/type d'établissements comparés) sont différents sur les deux périodes (Annexe A, Tableau A1). L'étude de 1994-95 repose sur tous les établissements du secteur public. Nous restreignons donc l'information sur la période 2006-2011 à un échantillon portant sur le même périmètre. Ensuite, le nombre de GHM est passé d'environ 800 groupes à plus de 2200 groupes. Enfin, les méthodes de calcul de coûts, basées sur la comptabilité analytique, ne sont pas totalement identiques entre les deux périodes, cela peut influencer la comparaison des variances. Sous le budget global (1994-95), le recueil des coûts ne s'appliquait qu'aux établissements du secteur public. A partir de 2006, l'ENCC a évolué pour intégrer une méthodologie commune aux établissements ex-DG et exOQN. Depuis, la méthodologie évolue pour affiner la mesure du coût : traitement de certaines charges (travaux sur le coût de l'investissement), recherche d'une plus grande discrimination des coûts (travaux en 2015 sur le recueil de la charge en soins) ${ }^{14}$.

Nous rapprochons nos résultats obtenus après la mise en place de la Tarification à l'activité (T2A) avec les valeurs qui ont été obtenues lorsque les établissements hospitaliers étaient tarifés par budget global (Annexe A). Ceci permet alors de mettre en regard les chiffres actuels avec ceux trouvés avant la mise en place de la T2A pour les établissements publics et ESPIC. La part de la variance inter-GHM des coûts représentait une très faible part de la variance totale $(20 \%)$ : la dispersion des coûts autour des valeurs moyennes était beaucoup plus importante. Même si aujourd'hui, il existe une certaine dispersion des coûts pour un même GHM, (de l'ordre de $67 \%$ pour les établissements publics et $79 \%$ pour les ESPIC) on observe tout de même une très forte concentration des coûts autour de la moyenne comparativement à la période sous budget global.

Ainsi, pour un GHM donné, les coûts semblent plus homogènes après la T2A qu'avant. Par ailleurs, la variance inter-GHM a augmenté au cours du temps quel que soit le type d'établissement public. Pour un GHM donné, les coûts sont de plus en plus homogènes au cours du temps quelle que soit la taille de l'établissement public ou ESPIC entre 2006 et 2011 (Annexe A, Tableau $\mathrm{A} 2$ ). Ceci est encore plus vrai pour les établissements à but lucratif.

\footnotetext{
${ }^{13}$ Nous nous sommes également inspirés de la méthodologie adoptée par l'équipe du projet EuroDRG, proche du papier Dormont et Milcent (2000).

14 L'ENCC est une information qui n'est pas construite sur des méthodes de microcosting mais sur des méthodes de comptabilité analytique. Source: Agence technique de l'information sur l'hospitalisation (ATIH), Responsable du Service en charge de la mise en place de l'ENCC.
} 
L'effort de réduction du coût est un effort fait par chaque hôpital quel que soit le séjour. Il est donc une composante des spécificités permanentes des établissements pour une année donnée. Supposons que le coût du séjour est une fonction décroissante de l'effort de réduction du coût. Par ailleurs, hypothèse forte, supposons que la part de variance due aux écarts permanents de coûts entre les établissements ne varie qu'en fonction de cet effort de réduction du coût. Cette hypothèse du modèle de Shleifer (1983), était empiriquement celle adoptée par Dormont et Milcent (2000). Nous comparons alors nos résultats avec ceux obtenus dans leur papier sur la période 1994-1995 pour les établissements du secteur public. Cette part de la variance interhôpitaux était de 9,5\%. Elle est ici de 12,1\%. Il est surprenant de voir que la variance interhôpitaux semble avoir légèrement augmenté avant et après la T2A (Tableau A2). Concernant la prise en charge d'IAM, depuis 2006, la politique de la concentration de la chirurgie cardiaque par seuils de volume d'activité a restructuré le marché hospitalier. Cette politique (anti-concurrence) peut également avoir un impact sur la variance inter-hôpitaux des coûts observés, expliquant ce résultat.

Afin de comparer ces résultats à ceux qui ont été obtenus par Dormont et Milcent (2000), nous incluons les actes et la durée de séjour dans le jeu des variables explicatives, après contrôle par la classification des GHM. En ne considérant que les établissements publics, la part additionnelle de variance expliquée par les caractéristiques du séjour du patient est de 40\%. Elle était d'environ 50\% sur la période 1994-1995. Avec les réserves précédemment évoquées de la comparabilité des périodes, il semble donc que la classification actuelle des GHM a permis de prendre en compte une plus grande part de la variance expliquée par les spécificités individuelles des séjours des patients.

\section{Conclusion et discussion}

Nous exploitons la base de données utilisée pour la fixation des tarifs de Groupe Homogène de Malades (GHM) sur les années 2006 à 2011. Cette base de données contient des informations précises sur le niveau de sévérité de l'état de santé d'un patient atteint d'infarctus du myocarde. Ces données sont collectées en routine. Par ailleurs, cette base fournit également le coût par séjour mesuré suivant la même comptabilité analytique par les établissements.

Ce papier est organisé autour de deux questions que nous nous proposons de reprendre ici.

Est-ce que la classification capture suffisamment l'hétérogénéité des coûts pour éviter les effets néfastes de sélection des patients ou de diminution du niveau de qualité que peut engendrer une tarification par forfait?

Les GHM ne captent qu'un tiers de la variance de coûts. Les hôpitaux étant rémunérés par forfait par GHM, ce résultat soulève la question de la prise en compte adéquate des coûts par les forfaits. Ces derniers, utilisés comme mode de financement ne permettent de prendre en compte qu'un tiers de l'ensemble de l'hétérogénéité des coûts des séjours par rapport à l'individu moyen. Sans prise en compte des GHM, 43\% de la variance des coûts sont expliquées par les caractéristiques individuelles des patients, les diagnostics et les procédures. Ainsi, les caractéristiques observables des séjours des patients expliquent davantage que la classification des GHM.

Une première étude de Dormont et Milcent (2000) étudiait l'effet potentiel d'un remboursement par forfait par séjour pour les établissements de santé encore tarifés par 
budget global. Il apparaissait de fortes variabilités de coûts entre les séjours d'un même GHM, notamment par une forte hétérogénéité des patients et une hétérogénéité des hôpitaux. Ceci remettait alors en question la validité de l'homogénéité de coûts au sein de chaque groupe (variance intra-GHM des coûts). Il était à craindre que la mise en place d'une tarification par forfait par séjour ait des effets pervers sur la qualité des pratiques des soins. En fait, nous montrons que sans parvenir à l'homogénéisation des coûts, une tendance à la baisse des hétérogénéités des coûts s'observe sur la période 2006-2011. Cette crainte est toujours de mise mais se réduit au cours du temps.

Comme souligné dans le texte, la comparabilité des périodes 1994-1995 et 2006-2011 comporte des limites indéniables. Dans un certains sens, ces limites peuvent être comparées à des études de comparaison de pays. Nous montrons que la réduction de l'hétérogénéité des coûts s'observe autant entre les périodes des deux études que sur la période 2006-2011.

Par ailleurs, nous montrons des disparités de variabilités de coûts par GHM. Suivant le statut de l'établissement, les coûts sont plus ou moins homogènes. Quelle que soit l'année considéré, les établissements privés présentent moins de différences de coûts que les autres statuts d'établissements. La comparaison temporelle suggère même un trend de plus grande homogénéisation des coûts par GHM pour les établissements privés que pour les autres statuts d'établissements. Ce résultat peut être dû à plus d'efficacité, d'homogénéité des pratiques ou d'homogénéité des caractéristiques des patients. Les données actuelles ne permettent pas de répondre plus avant à cette question. Une voie d'extension possible de ce travail serait d'obtenir des informations plus précises non seulement sur des variables de résultats comme les taux de réadmissions mais également sur des variables portant sur le processus de soins du patient.

La comparaison temporelle suppose ici que la représentativité des hôpitaux de la base ENCC avec la situation sur le territoire français soit respectée au cours du temps. Pour une comparaison entre secteurs, cette hypothèse doit également être vraie par secteur. Le montant des forfaits étant différent suivant le statut public ou privé des établissements, la tutelle fait face à cette contrainte dans la construction des bases ENCC. Toutefois, la faible participation des établissements privés à l'ENCC certaines années, peut remettre en cause cette hypothèse de représentativité temporelle de l'échantillon par secteur.

La seconde série de questions soulevée dans ce papier est : quelle est la part explicative de la variance du coût due aux écarts permanents de coûts moyens entre les établissements et s'explique-t-elle par les caractéristiques observables du séjour du patient ? Nous montrons que les spécificités permanentes des hôpitaux expliquent environ $20 \%$ de la variance de coût du séjour. Après prise en compte de la classification des GHM ainsi que des caractéristiques des patients, ces spécificités expliquent encore 5\% de la variance totale des coûts. Cette part de variance ne semble pas être due à des différences de durée de séjours.

Par ailleurs, après prise en compte de la classification des GHM et des caractéristiques des patients, le statut public, privé ou ESPIC des établissements de santé n'apportent pas d'information supplémentaire sur le coût du séjour.

L'analyse porte sur une pathologie, l'IAM. Les analyses portent donc sur une pathologie spécifique. Les résultats peuvent varier pour d'autres prises en charges (GHM). Le choix de cette pathologie permet à la fois des comparaisons internationales comme ceux de Hakkinen (2012) mais aussi dans le temps (Dormont et Milcent, 2000). Toutefois, une extension 
possible serait de réaliser cette étude sur d'autres pathologies.

\section{Bibliographie}

Bloom N., Cooper Z., Gaynor M., Gibbons S., Jones S., McGuire A., Moreno-Serra R., Propper C., Van Reenen J. et S. Seiler (2011), "In defence of our research on competition in England's National Health Service”, The Lancet, 378(9809), pp. 2064-2065.

Bradford WD, Kleit AN, Krousel-Wood MA, Re RN. 2001. Stochastic Frontier Estimation of Cost Models Within the Hospital. The Review of Economics and Statistics 83 : 302-309.

Brekke et Sogard, 2007. Public versus Private Health Care in a National Health Service. Health Economics 16 : 579-601

Cooper Z., Gibbons S., Jones S. and A. McGuire (2011), "Does hospital competition save lives? Evidence from the English patient choice reforms", The Economic Journal, 121, F228F260.

Chalkley et Malcomson 1998 Contracting for Health Services with unmonitored Quality. The Economic Journal 108

Chalkley et Malcomson 2000 Government purchasing of health services. Handbook on Health Economics, Chapter 15. Culyer AJ, Newhouse JP (eds). North Holland; Elsevier : Amsterdam

Dormont, B et Milcent C. (2000) "Coûts hospitaliers et tarification par pathologie : le cas de l'infarctus du myocarde aigu ", Revue d'Economie Politique, 110 (4)

Dormont B, Milcent C. 2005. How to regulate heterogeneous hospitals? Journal of Economics and Management Strategy 14 : 591-621.

Ellis et McGuire 1986 Provider Behavior under Prospective Reimbursement. Journal of health Economics 5 : 129-151

Ellis et McGuire 1990 Optimal payment systems for health services. Journal of Health Economics $9: 375-396$

Gaynor M. (2006), "Competition and Quality in Health Care Markets", Foundations and Trends in Microeconomics, 2(6), pp. 441-508.

Gaynor M., Moreno-Serra R. and C. Propper (2013), "Death by market power : reform, competition and patient outcomes in the National Health Service", American Economic Journal : Economic Policy, forthcoming.

Gobillon et Milcent (2014) Hospital Competition and Reimbursement System based on DRG, Working Paper PSE 
Gowrisankaran G. and R. Town (2003), "Competition, Payers, and Hospital Quality", Health Services Research, 38(6), pp. 1403-1422.

Hafsteinsdottir, E.J.G. and Siciliani, L. (2009). "DRG prospective payment systems : refine or not refine?" Health Economics, 19(10) : 1226-39.

Kessler D. and M. McClellan (2000), "Is hospital competition socially wasteful?", Quarterly Journal of Economics, 115(2), pp. 577-615.

Kessler D, McClellan M. 2002. The effects of hospital ownership on medical productivity. The Rand Journal of Economics 33 : 488-506.

Kessler D. and J. Geppert (2005), "The Effects of Competition on Variation in the Quality and Cost of Medical Care", Journal of Economics \& Management Strategy, 14(3), pp. 575589.

Laudicella M, Olsen KR, Street A. 2010. Examining cost variation across hospital departments - a two-stage multilevel approach using patient level data. Social Science \& Medicine 71 : 1872-1881.

Ma, CA. 1994 Health Care Payment Systems : Cost and Quality Incentives. Journal of Economics and Management Strategy 3 : 93-112

Mason A., Zeynep Or, Thomas Renaud, Andrew Street, Josselin Thuilliez et Padraic Ward on behalf of the EuroDRG Group "How well do diagnosis-related groups for Appendectomy explain variations in resource use? An analysis of patient-level data from 10 european Countries", Health Economics. 21(Suppl. 2) : 30-40 (2012)

McClellan M. 1997. Hospital reimbursement incentives : an empirical analysis. Journal of Economics and Management Strategy 6 : 91-128.

McClellan M. and D. Staiger (1999), "The Quality of Health Care Providers”, NBER Working Paper 7327.

Mougeot M. et Naegelen F., 2005 Hospital price regulation and expenditure cap policy. Journal of Health Economics 24 : 55-72

Olsen KR, Street A. 2008. The analysis of efficiency among a small number of organisations : how inferences can be improved by exploiting patient-level data. Health Economics 17 : 671281.

Pope G, 1989 Hospital Nonprice Competition and Medicare Reimbursement Policy. Journal of Health Economics 24 : 55-72

Shleifer A. (1985) "A theory of Yardstick competition", Rand Journal of Economics, 16 : 319-327

Street A, Kobel C, Renaud T, Thuilliez J. 2012. "How well do Diagnosis Related Groups explain variation in costs and length of stay among patients and across hospitals? Methods for analysing routine patient data". Health Economics 21 (Suppl. 2) : 6-18. 
Tableau 1 : La distribution des séjours et la distribution des hôpitaux

\begin{tabular}{|c|c|c|c|c|c|c|c|c|}
\hline \multirow[b]{4}{*}{ Public } & \multicolumn{7}{|c|}{ Distribution des séjours } & \multirow{3}{*}{$\begin{array}{l}\text { Période } \\
2006-2011 \\
\begin{array}{c}\text { Nombre de } \\
\text { séjours }\end{array}\end{array}$} \\
\hline & $\begin{array}{c}\text { Période } \\
\text { 2006-2011 }\end{array}$ & $\begin{array}{c}\text { Année } \\
2006\end{array}$ & $\begin{array}{c}\text { Année } \\
2007\end{array}$ & $\begin{array}{c}\text { Année } \\
2008 \\
\end{array}$ & $\begin{array}{c}\text { Année } \\
2009 \\
\end{array}$ & $\begin{array}{c}\text { Année } \\
2010\end{array}$ & $\begin{array}{l}\text { Année } \\
2011\end{array}$ & \\
\hline & $\%$ & $\%$ & $\%$ & $\%$ & $\%$ & $\%$ & $\%$ & \\
\hline & 82,3 & 63,0 & 66,2 & 86,7 & 89,7 & 88,8 & 90,5 & 43409 \\
\hline ESPIC & 8,5 & 10,02 & 9,92 & 7,76 & 8,76 & 7,57 & 6,76 & 4465 \\
\hline Privé & 9,2 & 26,96 & 23,86 & 5,5 & 1,49 & 3,69 & 2,71 & 4858 \\
\hline \multicolumn{9}{|c|}{ Nombre de séjours } \\
\hline $\begin{array}{l}\text { Tout } \\
\text { hôpitaux }\end{array}$ & 52732 & 6372 & 8250 & 8183 & 8185 & 10252 & 11490 & 52732 \\
\hline & \multicolumn{7}{|c|}{ Distribution des d'hôpitaux } & \\
\hline & $\begin{array}{c}\text { Période } \\
2006-2011\end{array}$ & $\begin{array}{l}\text { Année } \\
2006\end{array}$ & $\begin{array}{c}\text { Année } \\
2007\end{array}$ & $\begin{array}{c}\text { Année } \\
2008\end{array}$ & $\begin{array}{c}\text { Année } \\
2009\end{array}$ & $\begin{array}{c}\text { Année } \\
2010\end{array}$ & $\begin{array}{c}\text { Année } \\
2011\end{array}$ & 2011 \\
\hline & $\%$ & $\%$ & $\%$ & $\%$ & $\%$ & $\%$ & $\%$ & $\begin{array}{c}\text { Nombre } \\
\text { d'hôpitaux }\end{array}$ \\
\hline Public & $59,15 \%$ & $43,14 \%$ & $44,93 \%$ & $68,63 \%$ & $67,92 \%$ & $62,26 \%$ & $72,45 \%$ & 50 \\
\hline ESPIC & $17,68 \%$ & $21,57 \%$ & $20,29 \%$ & $19,61 \%$ & $15,09 \%$ & $15,09 \%$ & $13,83 \%$ & 18 \\
\hline Privé & $23,17 \%$ & $35,29 \%$ & $34,78 \%$ & $11,76 \%$ & $16,98 \%$ & $22,64 \%$ & $13,73 \%$ & 31 \\
\hline \multicolumn{9}{|c|}{ Nombre d'hôpitaux } \\
\hline $\begin{array}{l}\text { Tout } \\
\text { hôpitaux }\end{array}$ & 99 & 51 & 69 & 51 & 53 & 53 & 51 & 99 \\
\hline
\end{tabular}

Base ENCC, 52732 observations, 2006-2011 
Tableau 2 : Statistiques descriptives des séjours de patients

\begin{tabular}{|l|c|}
\hline Base ENCC & $\begin{array}{c}52 \text { 732 observations, } \\
\text { Années 2006-2011 }\end{array}$ \\
\hline Sexe & \\
Femme & $30,40 \%$ \\
Age & $67,24 \mathrm{ans}$ \\
Durée du séjour (DdS) & $6,45 \mathrm{jrs}$ \\
Coût total & $5890 €$ \\
Mode d'entrée du séjour & \\
D'un autre hôpital & $16,30 \%$ \\
Du domicile & $83,40 \%$ \\
Mode de sortie du séjour & \\
Vers un autre service & $2,0 \%$ \\
Vers un autre hôpital & $26,1 \%$ \\
Vers le domicile & $67,5 \%$ \\
Décédé & $4,3 \%$ \\
Procédures & \\
Stent & $64,41 \%$ \\
Angioplastie (sans stent) & $4,54 \%$ \\
Cathétérisme (sans angioplastie, stent) & \\
\hline Base ENCC, 52732 obser &
\end{tabular}

Base ENCC, 52732 observations, 2006-2011 
Tableau 3 : Statistiques descriptives des racines de GHM et des GHM

\begin{tabular}{|c|c|c|c|c|c|}
\hline & $\%$ & $\begin{array}{l}\text { Moyenne du } \\
\text { coût }(€)\end{array}$ & $\begin{array}{l}\text { Ecart-type du } \\
\text { coût }\end{array}$ & $\begin{array}{c}\text { Durée } \\
\text { moyenne } \\
\text { (jours) }\end{array}$ & $\begin{array}{l}\text { Ecart-type } \\
\text { de la durée }\end{array}$ \\
\hline \multicolumn{6}{|c|}{ Racine } \\
\hline $05 \mathrm{~K} 05$ & 54,1 & 6760,58 & 4825,66 & 6,20 & 4,50 \\
\hline $05 \mathrm{~K} 06$ & 9,1 & 5933,81 & 3746,42 & 5,07 & 4,36 \\
\hline 05M04 & 30,5 & 4868,37 & 4259,17 & 7,29 & 6,03 \\
\hline $05 \mathrm{M} 16$ & 6,4 & 3340,49 & 3948,48 & 6,54 & 5,87 \\
\hline \multicolumn{6}{|c|}{ GHM } \\
\hline $05 \mathrm{~K} 051$ & 35,9 & 5656,43 & 2342,01 & 4,76 & 2,64 \\
\hline $05 \mathrm{~K} 052$ & 13,5 & 7493,97 & 3259,16 & 7,79 & 4,59 \\
\hline $05 \mathrm{~K} 053$ & 4,0 & 11127,45 & 7483,90 & 11,50 & 6,63 \\
\hline 05K054 & 0,7 & 23848,12 & 22511,51 & 18,65 & 8,54 \\
\hline 05K061 & 5,7 & 5058,70 & 2318,00 & 3,79 & 2,28 \\
\hline 05K062 & 2,2 & 7176,15 & 3500,70 & 7,34 & 4,49 \\
\hline $05 \mathrm{~K} 063$ & 0,5 & 12222,66 & 6588,96 & 12,93 & 7,52 \\
\hline 05K064 & 0,1 & 15796,30 & 10760,95 & 15,07 & 6,62 \\
\hline $05 \mathrm{~K} 06 \mathrm{~T}$ & 0,6 & 3185,32 & 1267,59 & 1,00 & 0,00 \\
\hline $05 \mathrm{M} 041$ & 11,4 & 3579,57 & 2006,19 & 4,44 & 2,73 \\
\hline 05M042 & 8,3 & 5116,65 & 3200,75 & 8,00 & 5,09 \\
\hline $05 \mathrm{M} 043$ & 7,6 & 6615,82 & 4470,38 & 11,70 & 6,63 \\
\hline $05 \mathrm{M} 044$ & 0,9 & 13615,16 & 12472,04 & 16,38 & 8,73 \\
\hline $05 \mathrm{M} 04 \mathrm{~T}$ & 2,3 & 1404,41 & 981,92 & 1,00 & 0,00 \\
\hline 05M161 & 2,9 & 1615,39 & 1370,80 & 2,93 & 2,26 \\
\hline 05M162 & 1,5 & 3474,67 & 2593,62 & 7,33 & 5,13 \\
\hline $05 \mathrm{M} 163$ & 1,3 & 5117,51 & 4383,32 & 10,41 & 6,34 \\
\hline $05 \mathrm{M} 164$ & 0,7 & 7179,85 & 7472,72 & 13,05 & 6,47 \\
\hline
\end{tabular}

Base ENCC, 52732 observations, 328 hôpitaux 2006-2011

(Voir Annexe $C$ pour la signification des codes) 
Tableau 4 : Structure de la variance des coûts "Inter-GHM"

Les régressions sont contrôlées pour la tendance pour tenir compte de l'inflation

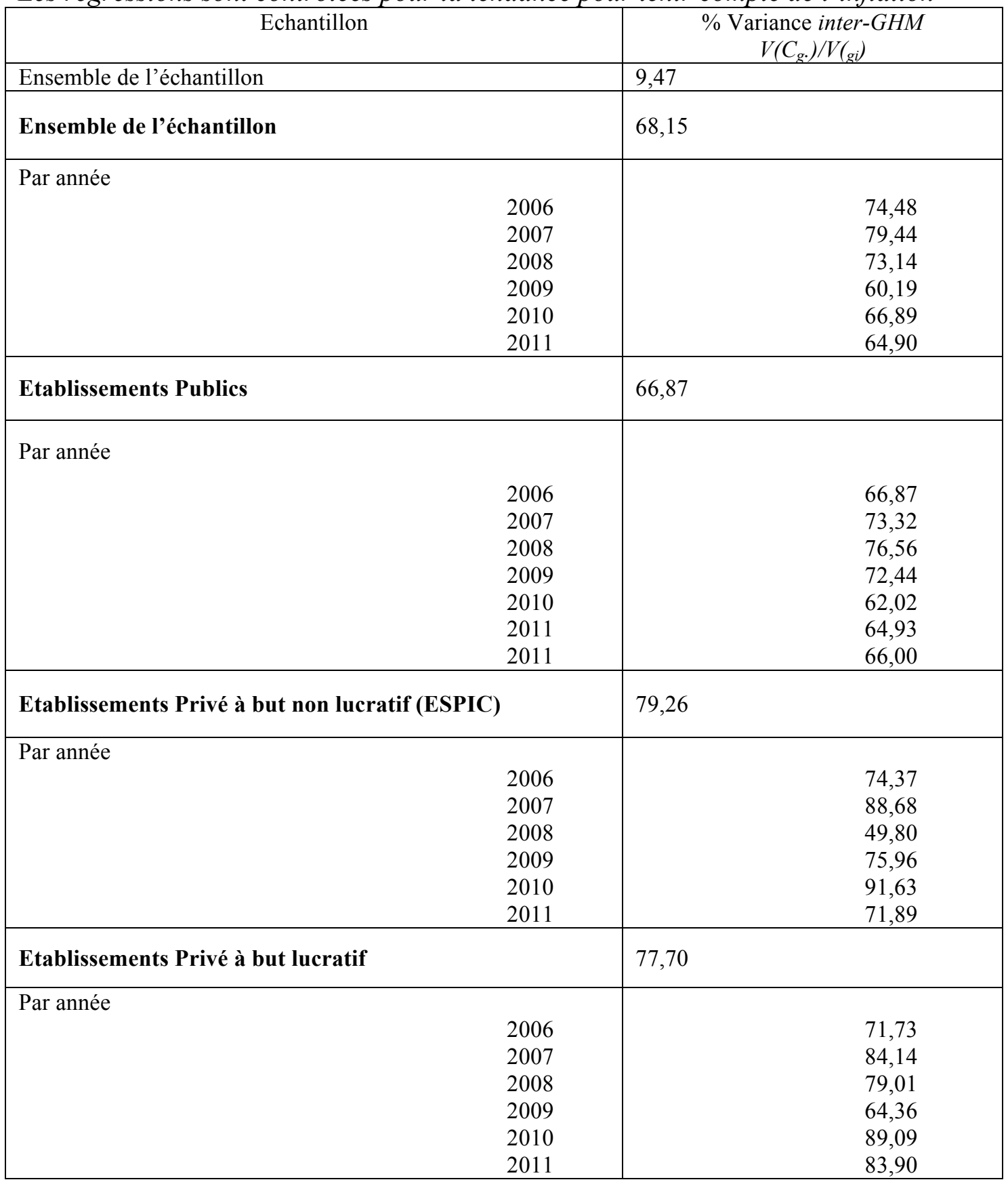

Base ENCC, 52732 observations, 2006-2011

ESPIC : Etablissements de santé à but non lucratif qualifiés d'établissements de santé privés d'intérêt collectif (ESPIC) 
Tableau 5 : Structure de la variance des coûts "Inter-hôpital"

Les regressions sont contrôlées pour la tendance pour tenir compte de l'inflation

\begin{tabular}{|c|c|}
\hline Echantillon & $\begin{array}{c}\text { \% Variance inter-hôpital } \\
V\left(C_{h} .\right) / V\left(C_{h i}\right)\end{array}$ \\
\hline $\begin{array}{l}\text { L'ensemble de } \\
\text { l'échantillon }\end{array}$ & 18,6 \\
\hline \multicolumn{2}{|l|}{ Par racine } \\
\hline 05K05 & 11,4 \\
\hline $05 \mathrm{~K} 06$ & 19,3 \\
\hline 05M04 & 20,1 \\
\hline $05 \mathrm{M} 16$ & 17,4 \\
\hline \multicolumn{2}{|l|}{ Par GHM } \\
\hline 05K051 & 26,5 \\
\hline $05 K 052$ & 21,0 \\
\hline $05 \mathrm{~K} 053$ & 13,0 \\
\hline $05 K 054$ & 23,3 \\
\hline 05K061 & 26,0 \\
\hline 05K062 & 25,3 \\
\hline $05 \mathrm{~K} 063$ & 30,7 \\
\hline 05K064 & 27,7 \\
\hline 05K06T & 26,7 \\
\hline 05М041 & 27,6 \\
\hline 05M042 & 21,1 \\
\hline $05 \mathrm{M} 043$ & 23,6 \\
\hline 05M044 & 22,6 \\
\hline 05M04T & 29,9 \\
\hline 05M161 & 19,2 \\
\hline $05 \mathrm{M} 162$ & 49,0 \\
\hline $05 \mathrm{M} 163$ & 27,7 \\
\hline 05M164 & 23,3 \\
\hline
\end{tabular}

Base ENCC, 52732 observations, 2006-2011

(Voir Annexe $C$ pour la signification des codes) 
Graphique 1 : Pourcentage de variance inter-hôpitaux par GHM

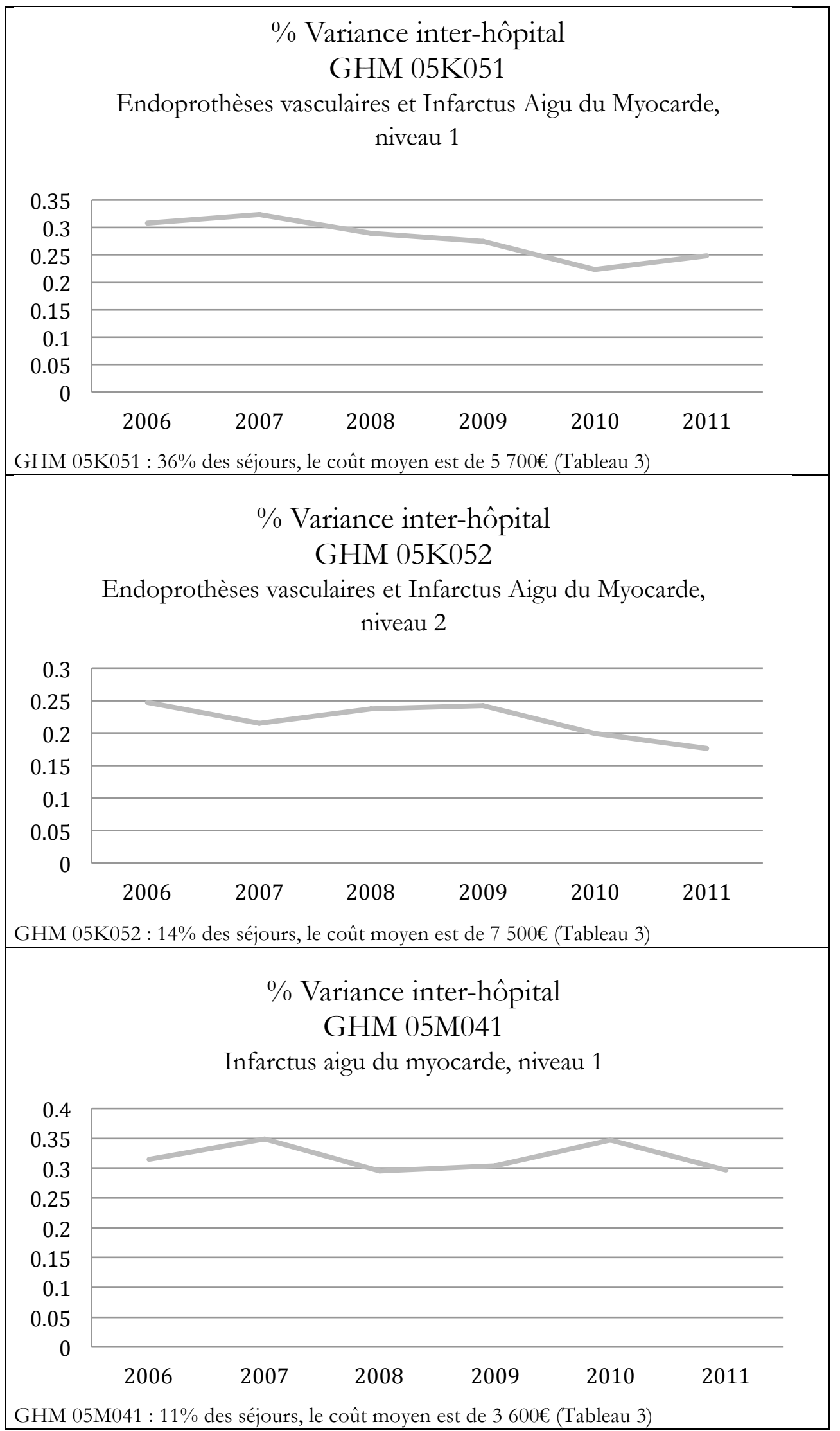

Base ENCC, 52732 observations, 2006-2011 
Graphique 2 : Pourcentage de variance de coût expliquée par les GHM sur la période 20062011

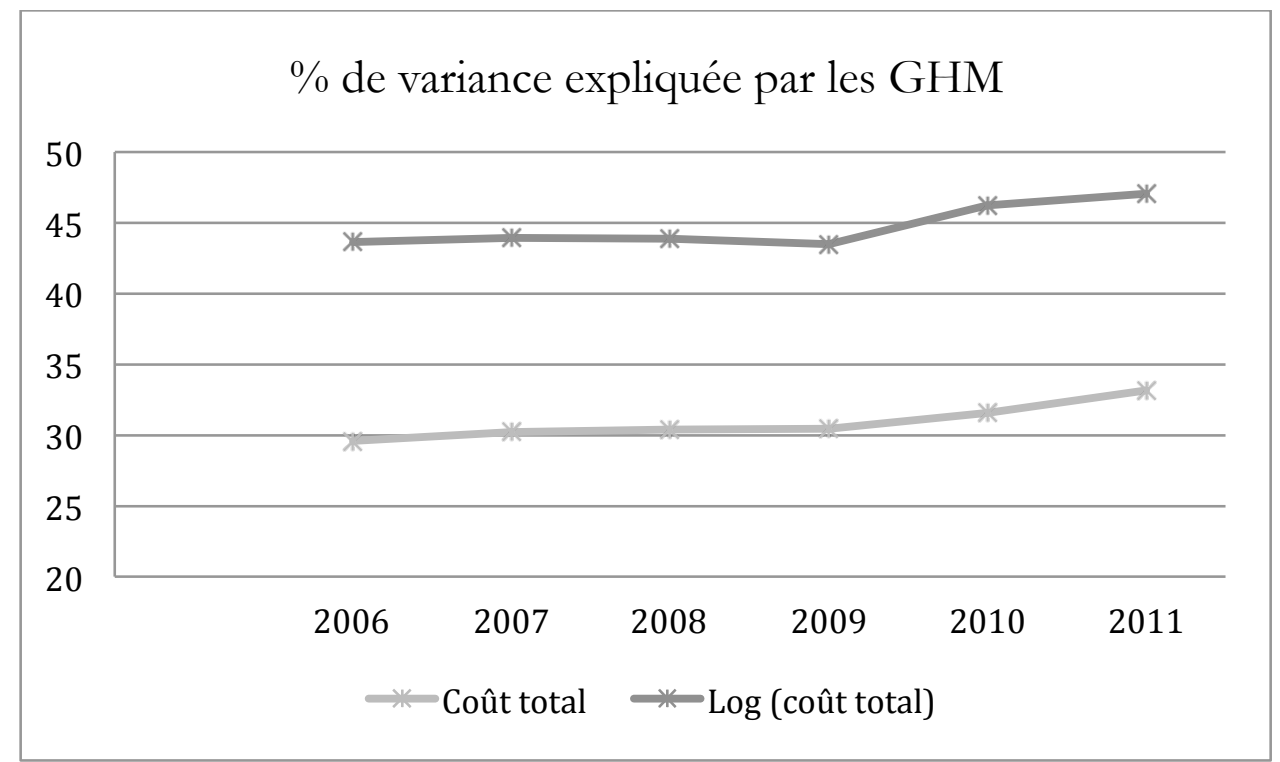

Base ENCC, 52732 observations, 2006-2011 
Tableau 6 : Estimation du coût total

\begin{tabular}{|c|c|c|c|c|c|}
\hline VARIABLES & $\log ($ Coût total $)$ & Coût total & VARIABLES & $\log ($ Coût total $)$ & Coût total \\
\hline & & & Trouble conduction et & $0.00826 * * *$ & $57.24 * * *$ \\
\hline Femme : 41-50 ans & $\begin{array}{l}-0.00383 \\
(0.0107)\end{array}$ & $\begin{array}{l}-75.79 \\
(90.53)\end{array}$ & rythme cardiaque & $(0.00194)$ & (16.44) \\
\hline Femme : $51-60$ ans & $\begin{array}{l}-0.0166^{*} \\
(0.00850)\end{array}$ & $\begin{array}{l}-27.50 \\
(72.09)\end{array}$ & Nombre de diag. & $\begin{array}{l}-0.00101^{*} \\
(0.000555)\end{array}$ & $\begin{array}{l}41.59 * * * \\
(4.709)\end{array}$ \\
\hline Femme : $61-70$ ans & $\begin{array}{l}-0.00295 \\
(0.00808)\end{array}$ & $\begin{array}{l}-114.5 * \\
(68.52)\end{array}$ & $05 \mathrm{~K} 052$ & $\begin{array}{l}0.0421 * * * \\
(0.00470)\end{array}$ & $\begin{array}{l}-122.1 * * * \\
(39.87)\end{array}$ \\
\hline Femme : $71-80$ ans & $\begin{array}{l}-0.0412 * * * \\
(0.00657)\end{array}$ & $\begin{array}{l}-335.6^{* * * *} \\
(55.73)\end{array}$ & $05 \mathrm{~K} 053$ & $\begin{array}{l}0.0646 * * * \\
(0.00779)\end{array}$ & $\begin{array}{l}384.2 * * * \\
(66.10)\end{array}$ \\
\hline Femme : 81-90 ans & $\begin{array}{l}-0.131 * * * \\
(0.00676)\end{array}$ & $\begin{array}{l}-765.2^{* * *} \\
(57.37)\end{array}$ & $05 \mathrm{~K} 054$ & $\begin{array}{l}-0.00869 \\
(0.0174)\end{array}$ & $\begin{array}{l}4,657 * * * \\
(147.7)\end{array}$ \\
\hline Femme : 91 ans et plus & $\begin{array}{l}-0.234 * * * \\
(0.0114)\end{array}$ & $\begin{array}{l}-1,054 * * * \\
(96.68)\end{array}$ & $05 \mathrm{~K} 061$ & $\begin{array}{l}-0.0769 * * * \\
(0.00661)\end{array}$ & $\begin{array}{l}-83.15 \\
(56.13)\end{array}$ \\
\hline Homme : 51-60 ans & $\begin{array}{l}-0.000944 \\
(0.00524)\end{array}$ & $\begin{array}{l}-7.482 \\
(44.45)\end{array}$ & $05 \mathrm{~K} 062$ & $\begin{array}{l}0.00232 \\
(0.00952)\end{array}$ & $\begin{array}{l}-166.6^{* *} \\
(80.76)\end{array}$ \\
\hline Homme : $61-70$ ans & $\begin{array}{l}-0.000366 \\
(0.00551)\end{array}$ & $\begin{array}{l}-23.78 \\
(46.73)\end{array}$ & $05 \mathrm{~K} 063$ & $\begin{array}{l}0.0375 * \\
(0.0197)\end{array}$ & $\begin{array}{l}509.0 * * * \\
(167.3)\end{array}$ \\
\hline Homme : $71-80$ ans & $\begin{array}{l}-0.0166 * * * \\
(0.00576)\end{array}$ & $\begin{array}{l}-157.5^{* * * *} \\
(48.88)\end{array}$ & $05 \mathrm{~K} 064$ & $\begin{array}{l}0.0786^{*} \\
(0.0470)\end{array}$ & $\begin{array}{l}1,979 * * * \\
(398.5)\end{array}$ \\
\hline Homme : 81-90 ans & $\begin{array}{l}-0.0940 * * * \\
(0.00682)\end{array}$ & $\begin{array}{l}-541.4^{* * *} \\
(57.84)\end{array}$ & $05 \mathrm{~K} 06 \mathrm{~T}$ & $\begin{array}{l}-0.280 * * * \\
(0.0191)\end{array}$ & $\begin{array}{l}-272.2^{*} \\
(162.4)\end{array}$ \\
\hline Homme : 91 ans et plus & $\begin{array}{l}-0.199 * * * \\
(0.0155)\end{array}$ & $\begin{array}{l}-1,095 * * * \\
(131.8)\end{array}$ & $05 \mathrm{M} 041$ & $\begin{array}{l}-0.397 * * * \\
(0.0364)\end{array}$ & $\begin{array}{l}-1,866^{* * * *} \\
(309.0)\end{array}$ \\
\hline Décès & $\begin{array}{l}0.0765 * * * \\
(0.00730)\end{array}$ & $\begin{array}{l}1,071 * * * \\
(61.96)\end{array}$ & $05 \mathrm{M} 042$ & $\begin{array}{l}-0.242 * * * \\
(0.0364)\end{array}$ & $\begin{array}{l}-2,122 * * * \\
(309.0)\end{array}$ \\
\hline Sortie : vers un autre service & $\begin{array}{l}-0.0407 * * * \\
(0.0104)\end{array}$ & $\begin{array}{l}-123.2 \\
(88.11)\end{array}$ & $05 \mathrm{M} 043$ & $\begin{array}{l}-0.178 * * * \\
(0.0366)\end{array}$ & $\begin{array}{l}-2,478 * * * \\
(310.2)\end{array}$ \\
\hline Sortie : vers un autre hôpital & $\begin{array}{l}-0.00643 * \\
(0.00348)\end{array}$ & $\begin{array}{l}210.5 * * * \\
(29.57)\end{array}$ & $05 \mathrm{M} 044$ & $\begin{array}{l}-0.116^{* * *} \\
(0.0392)\end{array}$ & $\begin{array}{l}-1,263 * * * \\
(332.7)\end{array}$ \\
\hline Entrée : d'un autre service & $\begin{array}{l}-0.127 * * * \\
(0.0315)\end{array}$ & $\begin{array}{l}-294.1 \\
(267.5)\end{array}$ & 05M04T & $\begin{array}{l}-0.932 * * * \\
(0.0377)\end{array}$ & $\begin{array}{l}-1,434 * * * \\
(319.9)\end{array}$ \\
\hline Entrée : d'un autre hôpital & $\begin{array}{l}-0.0877 * * * \\
(0.00422)\end{array}$ & $\begin{array}{l}-19.27 \\
(35.81)\end{array}$ & $05 \mathrm{M} 161$ & $\begin{array}{l}-0.893 * * * \\
(0.0375)\end{array}$ & $\begin{array}{l}-1,820 * * * \\
(318.2)\end{array}$ \\
\hline Alcool & $\begin{array}{l}-0.0119 * \\
(0.00645)\end{array}$ & $\begin{array}{l}13.79 \\
(54.76)\end{array}$ & $05 \mathrm{M} 162$ & $\begin{array}{l}-0.460 * * * \\
(0.0381)\end{array}$ & $\begin{array}{l}-2,261 * * * \\
(323.4)\end{array}$ \\
\hline Tabac & $\begin{array}{l}0.00886 * * * \\
(0.00265)\end{array}$ & $\begin{array}{l}-120.1 * * * \\
(22.47)\end{array}$ & $05 \mathrm{M} 163$ & $\begin{array}{l}-0.287 * * * \\
(0.0383)\end{array}$ & $\begin{array}{l}-2,387 * * * \\
(325.1)\end{array}$ \\
\hline Obésité & $\begin{array}{l}-0.000823 \\
(0.00335)\end{array}$ & $\begin{array}{l}-83.49 * * * \\
(28.43)\end{array}$ & $05 \mathrm{M} 164$ & $\begin{array}{l}-0.187 * * * \\
(0.0401)\end{array}$ & $\begin{array}{l}-2,188 * * * \\
(340.0)\end{array}$ \\
\hline Diabète & $\begin{array}{l}0.00596 * * * \\
(0.00231)\end{array}$ & $\begin{array}{l}-10.97 \\
(19.62)\end{array}$ & Stent & $\begin{array}{l}0.135 * * * \\
(0.0362)\end{array}$ & $\begin{array}{l}117.7 \\
(306.8)\end{array}$ \\
\hline Hypertension & $\begin{array}{l}0.00744 * * * \\
(0.00219)\end{array}$ & $\begin{array}{l}-80.69 * * * \\
(18.59)\end{array}$ & Cathétérisme & $\begin{array}{l}0.137 * * * \\
(0.00407)\end{array}$ & $\begin{array}{l}499.0 * * * \\
(34.56)\end{array}$ \\
\hline Problème reinaux & $\begin{array}{l}-0.00370 \\
(0.00311)\end{array}$ & $\begin{array}{l}-23.12 \\
(26.35)\end{array}$ & Angioplastie & $\begin{array}{l}0.266 * * * \\
(0.00698)\end{array}$ & $\begin{array}{l}1,079 * * * \\
(59.26)\end{array}$ \\
\hline Valvulopathie cardiaque & $\begin{array}{l}-0.00225 \\
(0.00354)\end{array}$ & $\begin{array}{l}-114.1 * * * \\
(30.02)\end{array}$ & $\mathrm{Nb}$ de procédures & $\begin{array}{l}0.00737 * * * \\
(0.000181)\end{array}$ & $\begin{array}{l}170.6 * * * \\
(1.537)\end{array}$ \\
\hline $\begin{array}{l}\text { Maladie artérielles } \\
\text { périphériques }\end{array}$ & $\begin{array}{l}0.00292 \\
(0.00372)\end{array}$ & $\begin{array}{l}-37.51 \\
(31.54)\end{array}$ & Durée du séjour & $\begin{array}{l}0.0696 * * * \\
(0.000378)\end{array}$ & $\begin{array}{l}489.8^{* * *} \\
(3.204)\end{array}$ \\
\hline Autre maladies vasculaires & $\begin{array}{l}-0.00774 \\
(0.00552)\end{array}$ & $\begin{array}{l}-196.5^{* * * *} \\
(46.88)\end{array}$ & Tendance & $\begin{array}{l}0.00872 * * * \\
(0.00107)\end{array}$ & $\begin{array}{l}26.17 * * * \\
(9.105)\end{array}$ \\
\hline Antécédent coronaire & $\begin{array}{l}-0.0181 * * * \\
(0.00472)\end{array}$ & $\begin{array}{l}-217.3 * * * \\
(40.04)\end{array}$ & Constante & $\begin{array}{l}8.058 * * * \\
(0.0376)\end{array}$ & $\begin{array}{l}2,415 * * * \\
(319.4)\end{array}$ \\
\hline \multirow[t]{2}{*}{ Insuffisante cardiaque } & $\begin{array}{l}-0.00397 \\
(0.00289)\end{array}$ & $\begin{array}{l}-32.15 \\
(24.54)\end{array}$ & & & \\
\hline & & & $\mathrm{R}^{2}$ & 0.769 & 0.7100 \\
\hline
\end{tabular}

Base ENCC, 52732 observations, 2006-2011

Standard errors in parentheses : *** $\mathrm{p}<0.01, * * \mathrm{p}<0.05, * \mathrm{p}<0.1$

(Voir Annexe $C$ pour la signification des codes) 
Tableau 7 : Part de la variance expliquée du coût total $\left(\mathrm{R}^{2}\right)$

\begin{tabular}{|c|c|c|c|}
\hline \multicolumn{2}{|c|}{ Modèles } & $\begin{array}{c}\text { Variable } \\
\text { expliquée } \\
C_{i g, h, t}: \\
\text { Coût total } \\
\end{array}$ & $\begin{array}{c}\text { Variable } \\
\text { expliquée } \\
C_{i g, h, t}: \\
\log (\text { coût total }) \\
\end{array}$ \\
\hline \multicolumn{4}{|c|}{ Modèle expliqué par les racines des GHM } \\
\hline & $C_{i, g, h, t}=\pi_{r, h, t}+\kappa T_{t}+u^{00}{ }_{i, g, h, t}$ & 5,67 & 18,75 \\
\hline \multicolumn{4}{|c|}{ Modèle expliqué par les niveaux de sévérités des GHM } \\
\hline & $C_{i, g, h, t}=\pi_{s, h, t}+\kappa T_{t}+u_{i, g, h, t}^{0}$ & 13,31 & 16,67 \\
\hline \multicolumn{4}{|c|}{ Modèles expliqués par les indicatrices des GHM, puis ajout successif de variables explicatives } \\
\hline A 1 & $C_{i, g, h, t}=\alpha_{g}+\kappa T_{t}+u_{i, g, h, t}^{l}$ & 30,15 & 45,11 \\
\hline \multirow[t]{2}{*}{2} & $C_{i, g, h, t}=\gamma \boldsymbol{X}_{i, g, h, t}+\alpha_{g}+\kappa T_{t}+u_{i, g, h, t}^{2}$ & 35,45 & 47,59 \\
\hline & Surcroît de variance dî̀ à: $X_{i, g, h, t}$ & $+5,3$ & $+2,48$ \\
\hline \multirow[t]{2}{*}{3} & $C_{i, g, h, t}=\boldsymbol{\beta}_{\boldsymbol{h}}+\gamma X_{i, g, h, t}+\alpha_{g}+\kappa T_{t}+u_{i, g, h, t}^{3}$ & 39,54 & 54,83 \\
\hline & Surcroît de variance dî̀ à: $\beta_{h}$ & $+4,09$ & $+7,24$ \\
\hline \multirow[t]{2}{*}{4} & 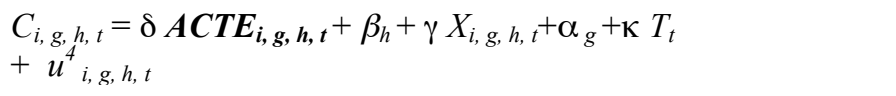 & 56,81 & 60,03 \\
\hline & Surcroît de variance dî à : $A C T E_{i, g, h, t}$ & $+17,27$ & $+5,2$ \\
\hline \multirow[t]{2}{*}{5} & $\begin{array}{l}C_{i, g, h, t}=\theta \boldsymbol{D} \boldsymbol{d} \boldsymbol{S}_{i, g, h, t}+\delta A C T E_{i, g, h, t}+\beta_{h}+\gamma X_{i, g, h, t}+\alpha_{g}+\kappa T_{t}+ \\
u_{i, g, h, t}^{g}\end{array}$ & 70,99 & 76,88 \\
\hline & Surcroît de variance dî̀ à : $D d S_{i, g, h, t}$ & $+14,18$ & $+16,85$ \\
\hline \multicolumn{4}{|c|}{ Modèles expliqués par les caractéristiques observables du patient durant le séjour sans puis avec les $G H M\left(\alpha_{g}\right)$} \\
\hline & $C_{i, g, h, t}=\delta A C T E_{i, g, h}+\gamma X_{i, g, h, t}+\kappa T_{t}+u_{i, g, h}^{6}$ & 43,18 & 39,13 \\
\hline & $C_{i, g, h, t}=\boldsymbol{\alpha}_{g}+\delta A C T E_{i, g, h}+\gamma X_{i, g, h, t}+\kappa T_{t}+u_{i, g, h, t}^{7}$ & 48,36 & 54,83 \\
\hline & Surcroît de variance dî̀ $\grave{a}: \alpha_{g}$ & $+5,18$ & $+15,70$ \\
\hline
\end{tabular}

Base ENCC, 52732 observations, 2006-2011

Avec $u^{k}$, les termes d'erreurs des modèles correspondants $(k=00,0$ puis de 1 à 7). Pour le modèle A5, l'estimation est biaisée si la durée du séjour ( $\left.D d S_{i g, h, t}\right)$ est non exogène

- $\quad \pi_{r, h, t}$ les variables indicatrices des racines $r$ de GHM pris en charge dans l'hôpital $h$, l'année $t$

- $\pi_{s, h, t}$ les variables indicatrices des niveaux de sévérité des GHM pris en charge dans l'hôpital $h$, l'année $t$ 
Tableau 8 : Rôle des spécificités permanents des hôpitaux $\left(\beta_{h}\right)$ dans la part de variance expliquée $\left(\mathrm{R}^{2}\right)$ du coût total

\begin{tabular}{|c|c|c|c|}
\hline & Modèles & $\begin{array}{c}\text { Variable } \\
\text { expliquée } \\
C_{i g, h, t}: \\
\text { Coût total }\end{array}$ & $\begin{array}{c}\text { Variable } \\
\text { expliquée } \\
C_{i g, h, t}: \\
\log (\text { coût total })\end{array}$ \\
\hline \multicolumn{4}{|c|}{ Modèle expliqué par les caractéristiques observables du séjour hors durée du séjour } \\
\hline \multirow{3}{*}{$\begin{array}{ll}C & 1 \\
& 2\end{array}$} & $C_{i, g, h, t}=\delta A C T E_{i, g, h, t}+\gamma X_{i, g, h, t}+\alpha_{g}+\kappa T_{t}+u_{i, g, h, t}^{7}$ & 48,36 & 54,83 \\
\hline & $\begin{array}{l}C_{i, g, h, t}=\boldsymbol{\beta}_{\boldsymbol{h}}+\delta A C T E_{i, g, h, t}+\gamma X_{i, g, h, t}+\alpha_{g}+\kappa T_{t} \\
+u_{i, g, h, t}^{8}\end{array}$ & 56,81 & 60,03 \\
\hline & $\begin{array}{l}\text { Surcrô̂t de variance dû aux spécificités permanentes des } \\
\text { hôpitaux : } \beta_{h}\end{array}$ & $+8,45$ & $+5,2$ \\
\hline \multicolumn{4}{|c|}{ Modèle expliqué par les caractéristiques observables du séjour dont durée du séjour } \\
\hline \multirow{3}{*}{$\begin{array}{ll}D & 1 \\
& \\
& 2\end{array}$} & $\begin{array}{l}C_{i, g, h, t}=\theta D d S_{i, g, h, t}+\delta A C T E_{i, g, h, t}+\gamma X_{i, g, h, t}+\alpha_{g} \\
+\kappa T_{t}+u_{i, g, h, t}^{9}\end{array}$ & 65,16 & 70,76 \\
\hline & $\begin{array}{l}C_{i, g, h, t}=\boldsymbol{\beta}_{\boldsymbol{h}}+\theta D d S_{i g, h, t}+\delta A C T E_{i g, h, t}+\beta_{h}+X_{i, g, h}{ }^{\prime} \gamma \\
+\kappa T_{t}+\alpha_{g}+u_{i, g, h, t}^{l 0}\end{array}$ & 70,99 & 76,88 \\
\hline & $\begin{array}{l}\text { Surcroît de variance dû aux spécificités permanentes des } \\
\text { hôpitaux : } \beta_{h}\end{array}$ & $+5,83$ & $+6,12$ \\
\hline \multicolumn{4}{|c|}{ Modèle expliqué par le statut des hôpitaux versus les spécificités permanents des hôpitaux } \\
\hline & $C_{i, g, h, t}=\gamma X_{i, g, h, t}+\alpha_{g}+\kappa T_{t}+u_{i, g, h, t}^{2}$ & 35,45 & 47,59 \\
\hline & $C_{i, g, h, t}=\zeta Z_{h}+\gamma X_{i, g, h, t}+\alpha_{g, h, t}+\kappa T_{t}+u_{i, g, h, t}^{l l}$ & 35,69 & 48,31 \\
\hline & Surcroît de variance dû au statut des hôpitaux : $Z_{h}$ & $+0,24$ & $+0,72$ \\
\hline & $C_{i, g, h, t}=\gamma X_{i, g, h, t}+\alpha_{g}+\kappa T_{t}+u_{i, g, h, t}^{2}$ & 35,45 & 47,59 \\
\hline & $C_{i, g, h, t}=\boldsymbol{\beta}_{\boldsymbol{h}}+\gamma X_{i, g, h, t}+\alpha_{g}+\kappa T_{t}+u_{i, g, h, t}^{3}$ & 39,54 & 54,83 \\
\hline & $\begin{array}{l}\text { Surcroît de variance dî aux spécificités permanentes des } \\
\text { hôpitaux: } \beta_{h}\end{array}$ & $+4,09$ & $+7,24$ \\
\hline
\end{tabular}

Base ENCC, 52732 observations, 2006-2011

Avec $u^{k}$, les termes d'erreurs des modèles correspondants $(k=1$ à 10). Pour le bloc de modèles D, les estimations sont biaisées si la durée du séjour $\left(D d S_{i, h, t}\right)$ est non exogène $Z_{h}$ : statut des hôpitaux - Public, ESPIC ou Privé à but lucrative ESPIC : Etablissements de santé à but non lucratif qualifiés d'établissements de santé privés d'intérêt collectif (ESPIC) 


\section{Annexe A : Compléments statistiques et économétriques}

Tableau A1 : base ENCC, années 2006-2011 et années 1994-1995

\begin{tabular}{|c|c|c|}
\hline Base ENCC & $\begin{array}{c}52732 \\
\text { observations, } \\
\text { Années 2006- } \\
2011\end{array}$ & $\begin{array}{c}5623 \\
\text { observations, } \\
\text { Années } \\
1994-1995\end{array}$ \\
\hline \multicolumn{3}{|l|}{ Sexe } \\
\hline Femme & $30,40 \%$ & $29,6 \%$ \\
\hline Age & 67,24 ans & \\
\hline ag40_64 & $44,5 \%$ & $39,8 \%$ \\
\hline ag65_89 & $51,8 \%$ & $56,5 \%$ \\
\hline ag90 & $3,8 \%$ & $3,7 \%$ \\
\hline Durée du séjour & $6,45 \mathrm{jrs}$ & \\
\hline Une journée & $5,5 \%$ & $6,9 \%$ \\
\hline Entre 2 et 5 jours & $48,6 \%$ & 2810 \\
\hline Entre 6 et 7 jours & $18,0 \%$ & $28,1 \%$ \\
\hline Entre 8 et 14 jours & $20,9 \%$ & $43,8 \%$ \\
\hline Plus de 14 jours & $7,0 \%$ & $21,2 \%$ \\
\hline \multicolumn{3}{|l|}{ Mode d'entrée du séjour } \\
\hline D'un autre hôpital & $16,30 \%$ & $7,2 \%$ \\
\hline Du domicile & $83,40 \%$ & $92,8 \%$ \\
\hline \multicolumn{3}{|l|}{ Mode de sortie du séjour } \\
\hline Vers un autre service & $2,0 \%$ & $3,4 \%$ \\
\hline Vers un autre hôpital & $26,1 \%$ & $26,8 \%$ \\
\hline Vers le domicile & $67,5 \%$ & $60,4 \%$ \\
\hline Décédé & $4,3 \%$ & $9,4 \%$ \\
\hline Coût total & $5890 €$ & \\
\hline \multicolumn{3}{|l|}{ Informations Générales } \\
\hline Nombre d'observations & 52732 & 5623 \\
\hline Nombre d'hôpitaux & 99 & \\
\hline Nombre de GHM & 13 & 6 \\
\hline Période & 2006-2011 & 1994-1995 \\
\hline
\end{tabular}

Pour les années 2006-2011, le mode d'entrée peut aussi être "autre service" du même hôpital 
Tableau A2 : \% Variance inter-GHM $V\left(C_{g .}\right) / V\left(C_{g i}\right)$

\begin{tabular}{|r|ccc|}
\hline \multirow{2}{*}{} & \multicolumn{3}{|c|}{ Statut de l'établissement } \\
& Public & ESPIC & Privé \\
\hline & \% Variance & inter-hôpital $V\left(C_{h}.\right) / v\left(C_{h i}\right)$ \\
\hline Toutes années & $12,1 \%$ & 10,2 & 28,1 \\
\hline & \% Variance & inter-GHM $V\left(C_{g}\right) / V\left(C_{g i}\right)$ \\
\hline Toutes années & 66,87 & 79,26 & 77,70 \\
\hline 2006 & 73,32 & 74,37 & 71,73 \\
2007 & 76,56 & 88,68 & 84,14 \\
2008 & 72,44 & 49,80 & 79,01 \\
2009 & 62,02 & 75,96 & 64,36 \\
2010 & 64,93 & 91,63 & 89,09 \\
2011 & 66,00 & 71,89 & 83,90 \\
\hline
\end{tabular}

Base ENCC, 52732 observations, 2006-2011

ESPIC : Etablissements de santé à but non lucratif qualifié d'établissements de santé privés d'intérêt collectif

Tableau A3 : \% Variance inter-GHM $V\left(C_{g .}\right) / V\left(C_{g i}\right)$

\begin{tabular}{|c|c|c|c|}
\hline & Modèles & $\begin{array}{c}\text { Variable } \\
\text { expliquée } \\
C_{i g, h, t}: \\
\text { Coût total }\end{array}$ & $\begin{array}{c}\text { Variable expliquée } \\
C_{i g, h, t}: \\
\log (\operatorname{coût} \text { total })\end{array}$ \\
\hline $\bar{A}$ & $\begin{array}{l}C_{i g, h, t}=\boldsymbol{\alpha}_{g}+\kappa T_{t}+u_{i, g, h, t}^{l} \\
C_{i g, h, t}=\gamma \boldsymbol{X}_{i, g, h, t}+\alpha_{g}+\kappa T_{t}+u_{i, g, h, t}^{2} \\
C_{i g, h, t}=\boldsymbol{\beta}_{h}+\gamma X_{i g, h, t}+\alpha_{g}+\kappa T_{t}+u_{i, g, h, t}^{3} \\
C_{i g, h, t}=\delta \boldsymbol{A} \boldsymbol{A T C} \boldsymbol{E}_{i, g, h, t}+\beta_{h}+\gamma X_{i g, h, t}+\alpha_{g}+\kappa T_{t}+u_{i, g, h, t}^{4} \\
C_{i g, h, t}=\theta \boldsymbol{D} \boldsymbol{d} \boldsymbol{S}_{i, g, h, t}+\delta A C T E_{i, g, h, t}+\beta_{h}+\gamma \boldsymbol{X}_{i g, h, t}+\alpha_{g}+\kappa T_{t}+ \\
u_{i, g, h, t}^{5}\end{array}$ & $\begin{array}{l}\mathbf{3 0 , 1 5} \\
35,45 \\
39,54 \\
56,81 \\
\mathbf{7 0 , 9 9}\end{array}$ & $\begin{array}{l}\mathbf{4 5 , 1 1} \\
47,59 \\
54,83 \\
60,03 \\
\mathbf{7 6 , 8 8}\end{array}$ \\
\hline$B$ & $\begin{array}{l}C_{i g, h, t}=\boldsymbol{\alpha}_{g}+\kappa T_{t}+u_{i, g, h, t}^{l} \\
C_{i g, h, t}=\gamma \boldsymbol{X}_{i g, h, t}+\alpha_{g}+\kappa T_{t}+u_{i, g, h, t}^{2} \\
C_{i g, h, t}=\theta \boldsymbol{D} \boldsymbol{d} \boldsymbol{S}_{i, g, h, t}+\gamma X_{i g, h, t}+\alpha_{g}+\kappa T_{t}+v_{i, g, h, t}^{l} \\
C_{i g, h, t}=\boldsymbol{\beta}_{\boldsymbol{h}}+\theta D d S_{i, g, h, t}+\gamma X_{i g, h, t}+\alpha_{g}+\kappa T_{t}+v_{i, g, h, t}^{2} \\
C_{i g, h, t}=\delta \boldsymbol{A C T E} \boldsymbol{E}_{i, g, h, t}+\beta_{h}+\theta D d S_{i, g, h, t}+\gamma X_{i g, h, t}+\alpha_{g}+\kappa T_{t} \\
+u_{i, g, h, t}\end{array}$ & $\begin{array}{l}\mathbf{3 0 , 1 5} \\
35,45 \\
59,19 \\
62,92 \\
\mathbf{7 0 , 9 9}\end{array}$ & $\begin{array}{l}\mathbf{4 5 , 1 1} \\
47,59 \\
69,01 \\
75,45 \\
\mathbf{7 6 , 8 8}\end{array}$ \\
\hline $\bar{C}$ & $\begin{array}{l}C_{i g, h, t}=\boldsymbol{\alpha}_{g}+\kappa T_{t}+u_{i, g, h, t}^{l} \\
C_{i g, h, t}=\boldsymbol{X}_{i g, h, t}{ }^{t} \gamma^{2} \alpha_{g}+\kappa T_{t}+u_{i, g, h, t}^{2} \\
C_{i g, h, t}=\delta \boldsymbol{A C T E} \boldsymbol{E}_{i g, h, t}+\gamma X_{i g, h, t}+\alpha_{g}+\kappa T_{t}+v_{i, g, h, t}^{3} \\
C_{i g, h, t}=\boldsymbol{\beta}_{\boldsymbol{h}}+\delta A C T E_{i g, h, t}+\gamma X_{i g, h, t}+\alpha_{g}+\kappa T_{t}+u_{i, g, h, t} \\
C_{i g, h, t}=\theta \boldsymbol{D} \boldsymbol{d} \boldsymbol{S}_{i, g, h, t}+\beta_{h}+\delta A C T E_{i, g, h, t}+\gamma X_{i g, h, t}+\alpha_{g}+\kappa T_{t}+ \\
u_{i, g, h, t}^{5}\end{array}$ & $\begin{array}{l}\mathbf{3 0 , 1 5} \\
35,45 \\
48,36 \\
56,81 \\
\mathbf{7 0 , 9 9}\end{array}$ & $\begin{array}{l}\mathbf{4 5 , 1 1} \\
47,59 \\
54,83 \\
60,03 \\
\mathbf{7 6 , 8 8}\end{array}$ \\
\hline$\overline{D D}$ & 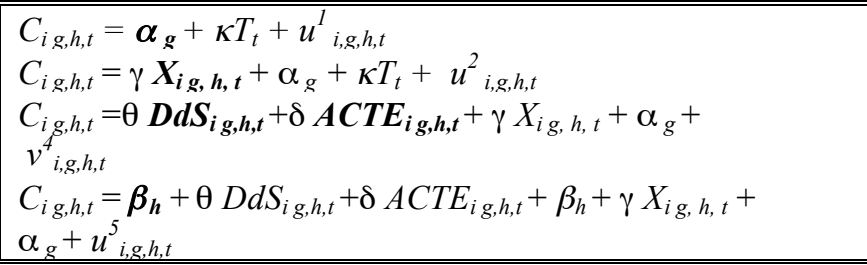 & $\begin{array}{l}\mathbf{3 0 , 1 5} \\
35,45 \\
65,16 \\
\\
\mathbf{7 0 , 9 9}\end{array}$ & $\begin{array}{l}\mathbf{4 5 , 1 1} \\
47,59 \\
70,76 \\
\\
\mathbf{7 6 , 8 8}\end{array}$ \\
\hline
\end{tabular}

Base ENCC, 52732 observations, 2006-2011

Avec $u^{k}$ et $v^{j}$, les termes d'erreurs des modèles correspondants $(k=1 \grave{a} 5$ et $j=1 \grave{a} 4)$. Les estimations sont biaisées si la durée du séjour $\left(D d S_{i g, h, t}\right)$ est non exogène 


\section{Annexe B : Classification version 11}

La classification des GHM a évolué à travers le temps. En 2004 et avec l'utilisation des GHM comme base de paiement, la classification retenue comportait environ 800 groupes. Chaque année, des modifications étaient réalisées à la marge, afin de prendre en compte des changements de prise en charge médicale du patient ainsi que des évolutions thérapeutiques éventuelles.

En 2009, le nombre de groupes est presque multiplié par 3 (près de 2200 GHM). Il s'agit du passage de la version 10 à la version 11. Ce changement s'accompagne d'un changement de logique dans la construction des GHM.

La classification des GHM est organisée en "poupée russe ». Ils sont codés sur 6 caractères. Les deux premiers caractères définissent la pathologie, le troisième caractère définit le type de pratique médicale (médicale, chirurgical ou exploratoire), les quatrième et cinquième caractères définissent les sous-champs d'une pathologie donnée. Le sixième caractère définit le niveau de sévérité. Il existe 4 niveaux de sévérité. La racine d'un GHM correspond aux 5 premiers caractères du GHM. Il s'agit du tronc commun. Il existe environ 600 racines. Ainsi, on passe d'une logique de classification privilégiant le nombre d'observations par groupes à une classification privilégiant l'homogénéité des groupes.

L'affectation d'un séjour dans un GHM donné se fait à partir de l'algorithme correspondant à la version des GHM en cours. A partir des diagnostics observés sur un patient, des actes réalisés sur le patient, du diagnostic principal du patient, un arbre de décisions permet l'association d'un séjour à un et unique GHM. Ces informations -ensemble des diagnostics, actes et du diagnostic principal ainsi que le GHM associé- sont contenues dans des bases appelées bases PMSI.

Cette classification version 11 définit quatre niveaux de sévérité. Le niveau de sévérité est indépendant de l'acte réalisé sur le patient.

- Niveau 1 : séjours sans complication ou morbidité associée (CMA) appartenant à la liste entérinée par les instances consultatives et décisionnelles de l'Agence Technique de l'Information sur l'Hospitalisation (ATIH). Les critères d'inclusion d'un CMA dans la liste sont entre autres, une augmentation de la durée de séjour d'au moins 2 jours et d'au moins $25 \%$ de la durée de référence ;

- Niveau 2, 3 ou $4:$ La liste des CMA contient trois sous-listes correspondant à trois niveaux de sévérité. Pour une version de la classification donnée, une CMA appartient toujours à un seul niveau de sévérité et n'en change pas. Dans certains cas, le décès peut également jouer le rôle de CMA. Dans l'algorithme de groupage, l'ordre de priorité est le suivant

- Existence d'une CMA et son appartenance à un niveau donné $(2,3$ ou 4$)$;

- Age du patient (selon le cas : $<2$ ans, $>69$ ans ou $>79$ ans) ;

- Décès si aucune des deux conditions précédentes n'est satisfaite ;

- Durée du séjour qui est une condition obligatoire.

D'une part, certaines racines de GHM ne présentent pas de subdivision en quatre niveaux de sévérité, voire même ne présente aucune subdivision en sévérité. D'autre part, il existe d'autres types de subdivisions qui remplacent ou complètent la subdivision en sévérité.

- La lettre "Z" indique l'absence de niveau de sévérité ;

- La lettre "E", pour les séjours se terminant par un décès ; 
- La lettre "J" identifie les séjours strictement ambulatoires (pas de nuit). Il s'agit de séjours chirurgicaux mais également de techniques interventionnelles.

- La lettre "T" correspond à des courts séjours, entre 1 et 3 jours. Il faut les distinguer des séjours codés "J". D'après l'Agence Technique d'Information Hospitalière (ATIH), il s'agit très souvent de séjours médicaux.

Les séjours pour infarctus aigus du myocarde sont répartis dans 35 racines différentes et 113 Groupes Homogènes de Malades (GHM). Une grande majorité des groupes regroupent moins de $1 \%$ des séjours pour IAM. Cinq racines regroupent un pourcentage de séjours supérieur à $1 \%$. Seuls 13 GHM ont plus de $1 \%$ de séjours.

L'échantillon retenu est composé des séjours admis pour infarctus aigus du myocarde et associé à un des GHM des 4 racines sélectionnées. ${ }^{15}$

15 Le Tableau 3 donne la répartition pour chaque GHM et de chaque racine de GHM dans l'échantillon. 


\title{
Annexe C : Catalogue des codes et leur signification pour les GHM et les racines de GHM
}

\author{
Catalogue des codes et leur signification pour les GHM
}

\begin{tabular}{|ll|}
\hline $05 \mathrm{~K} 051$ & Endoprothèses vasculaires avec infarctus du myocarde, niveau 1 \\
$05 \mathrm{~K} 052$ & Endoprothèses vasculaires avec infarctus du myocarde, niveau 2 \\
$05 \mathrm{~K} 053$ & Endoprothèses vasculaires avec infarctus du myocarde, niveau 3 \\
$05 \mathrm{~K} 054$ & Endoprothèses vasculaires avec infarctus du myocarde, niveau 4 \\
$05 \mathrm{~K} 051$ & Endoprothèses vasculaires avec infarctus du myocarde, niveau 1 \\
$05 \mathrm{~K} 052$ & Endoprothèses vasculaires avec infarctus du myocarde, niveau 2 \\
$05 \mathrm{~K} 053$ & Endoprothèses vasculaires avec infarctus du myocarde, niveau 3 \\
$05 \mathrm{~K} 054$ & Endoprothèses vasculaires avec infarctus du myocarde, niveau 4 \\
$05 \mathrm{~K} 061$ & Endoprothèses vasculaires sans infarctus du myocarde, niveau 1 \\
$05 \mathrm{~K} 062$ & Endoprothèses vasculaires sans infarctus du myocarde, niveau 2 \\
$05 \mathrm{~K} 063$ & Endoprothèses vasculaires sans infarctus du myocarde, niveau 3 \\
$05 \mathrm{~K} 064$ & Endoprothèses vasculaires sans infarctus du myocarde, niveau 4 \\
$05 \mathrm{~K} 06 \mathrm{~T}$ & Endoprothèses vasculaires sans infarctus du myocarde, très courte durée \\
$05 \mathrm{M} 041$ & Infarctus aigu du myocarde, niveau 1 \\
$05 \mathrm{M} 042$ & Infarctus aigu du myocarde, niveau 2 \\
$05 \mathrm{M} 043$ & Infarctus aigu du myocarde, niveau 3 \\
$05 \mathrm{M} 044$ & Infarctus aigu du myocarde, niveau 4 \\
$05 \mathrm{M} 04 \mathrm{~T}$ & Infarctus aigu du myocarde, très courte durée \\
$05 \mathrm{M} 161$ & Athérosclérose coronarienne, niveau 1 \\
$05 \mathrm{M} 162$ & Athérosclérose coronarienne, niveau 2 \\
$05 \mathrm{M} 163$ & Athérosclérose coronarienne, niveau 3 \\
$05 \mathrm{M} 164$ & Athérosclérose coronarienne, niveau 4 \\
\hline
\end{tabular}

\section{Catalogue des codes et leur signification pour les racines des GHM}

\begin{tabular}{|ll|}
\hline $05 \mathrm{~K} 05$ & Endoprothèses vasculaires avec infarctus du myocarde \\
$05 \mathrm{~K} 06$ & Endoprothèses vasculaires sans infarctus du myocarde \\
$05 \mathrm{M} 04$ & Infarctus aigu du myocarde \\
$05 \mathrm{M} 16$ & Athérosclérose coronarienne \\
\hline
\end{tabular}

La sélection des séjours pour Infarctus Aigu du Myocarde, a été effectuée par un médecin de l'ATIH responsable de la constitution de la base utilisée pour cette étude. Les séjours peuvent être affectés aux GHM pré-cités, incluant la racine 05K06. A titre d'exemple, les séjours ayant le diagnostic principal I2148 : "Infarctus sous-endocardique (infarctus du myocarde), autres prises en charge" est majoritairement associé au GHM 05K061 et de façon moindre, au GHM 05K051. Pour d'autres cas, le lecteur intéressé peut consulter les sites suivants (non exclusifs) :

- http://www.aideaucodage.fr/cim-i2148;

- $\quad$ http://www.has-sante.fr/portail/upload/docs/application/pdf/201402/endoprothese_coronaire_-_problematique_pertinence.pdf 


\section{Annexe D : Origine des forfaits}

Aux Etats-Unis, le tissu hospitalier est historiquement très majoritairement privé. La mise en place d'un gel des budgets hospitaliers comparable à la politique de budget global en France et plus globalement en Europe, n'y était pas possible. Les établissements de santé privés n'étant pas soumis à une mission de service public, ceux-ci étaient en mesure de refuser les patients dont le mode de remboursement ne semblait pas rentable aux gestionnaires. Ceci explique la mise en place précoce aux Etats-Unis d'une tarification basée sur un système d'information. Des groupes de pathologies ayant pour objectif d'être homogènes en coût et en diagnostics ont été construits. Des forfaits tarifaires sont alors calculés pour chacun des groupes. Tout séjour hospitalier est associé à un groupe et est remboursé sur la base du forfait associé à ce groupe. Appelés Diagnosis Related Groups (DRGs), ces groupes ont été utilisés dès 1983, pour le remboursement des patients de plus de 65 ans, pris en charge par le programme Medicare aux Etats-Unis.

Ce système de paiement a par la suite été adopté par des organismes d'assurance privés aux Etats-Unis, comme dans un grand nombre de pays développés. En France, durant cette période (1983-2003), un système d'information de l'activité hospitalière a été développé puis mis en place. Des groupes, Groupe Homogène de Malade (GHM) ${ }^{16}$ s'inspirant très largement de la construction des DRG ont été définis. A chaque séjour est associé un GHM unique. A chaque GHM est associé un forfait.

\footnotetext{
${ }^{16}$ De façon légèrement abusive, nous confondrons ici le groupe homogène de malades (GHM) avec le groupe homogène de séjours (GHS). Un GHM correspond à un ou plusieurs GHS. Celui-ci introduit une notion tarifaire en rapport avec l'utilisation du PMSI pour le financement des établissements de santé (tarification à l'activité).
} 